\title{
Scalable Synthesis of 1,3,4,5-Tetraaryl Imidazolium Salts as Precursors of Sterically Demanding $\mathbf{N}$-Heterocyclic Carbenes
}

\author{
Cengiz Azap ${ }^{\mathrm{a}, 1}$ \\ Anna Christoffers ${ }^{\mathrm{a}, 2}$ \\ Renat Kadyrov*a,b (i) \\ a EVONIK Resource Efficiency GmbH, Rodenbacher Chaussee 4, \\ 63457 Hanau, Germany \\ ${ }^{b}$ Institute of Inorganic Chemistry, Academy of Sciences of the \\ Czech Republic, 25068 Řež, Czech Republic \\ kadyrov@iic.cas.cz
}

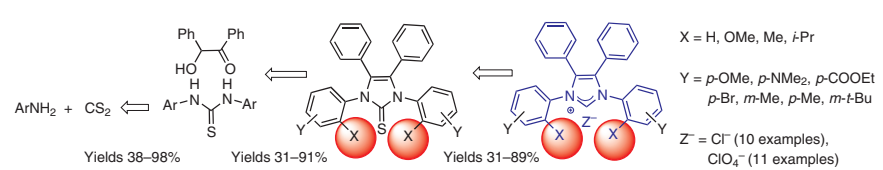

larly, QSAR analysis shows that imidazolylidene ligands with the backbone carbon atoms substituted by phenyl groups most efficiently promote catalytic activity of second-generation Ru catalysts in olefin metathesis. ${ }^{4}$ As a part of our efforts to provide access to effective metathesis catalysts, we were interested in a cost-effective and easily scalable synthesis of 1,3,4,5-tetraaryl imidazolium salts. ${ }^{5}$ Especially, we are seeking methods and procedures that are applicable industrially, avoiding chromatographic separation.

Over the past decades, many synthetic methods for the preparation of imidazolium salts have been reported. ${ }^{6,7} \mathrm{Al}$ though, the first synthesis of a tetraarylimidazolium salt was established almost 50 years ago, examples of 4,5-diaryl-substituted imidazolium salts are rare. ${ }^{8}$ Recently, one highly sterically demanding example $(\mathrm{Ar}=\mathrm{Mes})$ was reported (Scheme 1, A). ${ }^{8 d}$ Most recently, an aldimine coupling followed by cyclization with formaldehyde was used for the preparation of a wide variety of 4,5-diaryl-substituted NHCs with para-substituted $N$-phenyl groups (Scheme 1, B). ${ }^{8 e, f}$ An interesting method for formation of $1,3,4,5-$ tetraarylimidazoles is reaction of benzyl dianil with sodium in diethyl ether followed by addition of carbon disulfide (Scheme 1, C).8g

Unfortunately, protocols $A$ and $C$ are not really scalable and protocol $\mathrm{B}$ is restricted to the preparation of imidazolium salts with para-substituents on the $\mathrm{N}$-aryl groups. The erable attention as alternatives for widely used phosphine complexes in homogeneous catalysis. ${ }^{3}$ Among them, sterically demanding and electron-rich carbenes have been successfully utilized in most catalytic transformations. Particu-

Key words carbene ligands, heterocycles, imidazolium salts, imidazolinthiones, thioureas

N-Heterocyclic carbenes (NHCs) have attracted consid-

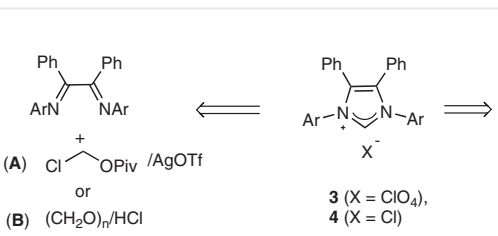

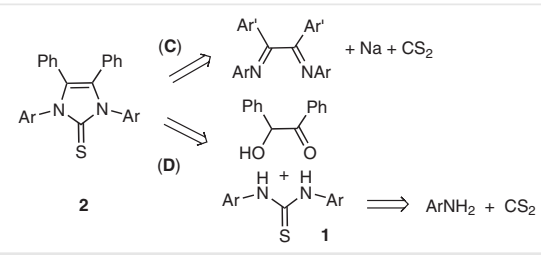

Scheme 1 Routes to 1,3,4,5-tetraarylimidazolium salts 


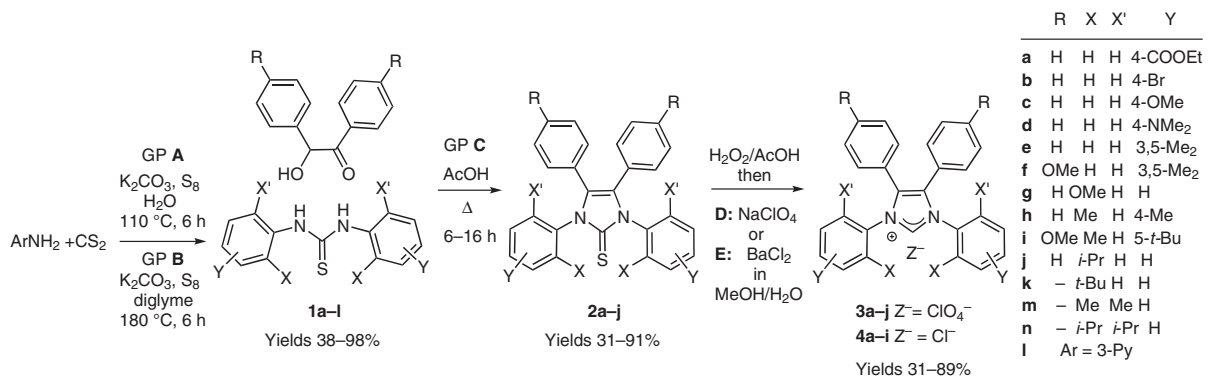

Scheme 2 Synthesis of 1,3,4,5-tetraarylimidazolium salts used in this work (route D)

strategy outlined in Scheme 2 appears to be the most convenient synthetic route for the large-scale and cost-efficient preparation of 4,5-diaryl-substituted imidazolium salts with sterically demanding $\mathrm{N}$-aryl groups.

A number of synthetic protocols to access 1,3-disubstituted thioureas 1 have been reported. ${ }^{9}$ Past approaches include reaction of thiophosgene, ${ }^{10}$ isothiocyanates, ${ }^{11}$ and $1,1^{\prime}$-thiocarbonyldiimidazole ${ }^{12}$ with amines. Recently, the direct conversion of aniline and $\mathrm{CS}_{2}$ into thiourea 1 has been developed. ${ }^{9 b, 13}$ After initial experiments, applying different published approaches, we found that $\mathrm{N}, \mathrm{N}$-diarylthioureas $1 \mathbf{c}-\mathbf{i}$ and $1 \mathbf{m}$ could be generated in good yields by the reaction of the amine with the $\mathrm{CS}_{2}$ in water; whereas heating under reflux for $6 \mathrm{~h}$ in diglyme was required for the formation of thioureas $\mathbf{1} \mathbf{j}$ and $\mathbf{1 k}$ bearing isopropyl- and tert-butyl groups at the ortho-positions, respectively. Furthermore, thioureas $\mathbf{1 a}, \mathbf{1 b}$, and $\mathbf{1 n}$ were obtained in better yields by using the latter method. The substitution patterns of the $N$-aryl groups were selected on the basis of availability of the corresponding anilines on large scale, including electron-rich (c, d, e) and with increased steric demand $(\mathbf{g}-\mathbf{k}, \mathbf{m}, \mathbf{n})$ substrates.

It should be noted at this point that only benzoin and anisoin are commercially available; therefore, no more benzoins were used in this survey. To introduce other substitution patterns into the aryl-groups at positions 4 and 5 requires significant synthetic effort. ${ }^{14}$

Imidazolin-2-thiones were obtained in good to excellent yields by condensation of the $\mathrm{N}, \mathrm{N}$-diarylureas with benzoin or anisoin in acetic acid, ${ }^{15}$ although the imidazolin-2-thione $\mathbf{2 j}$, bearing an isopropyl-group at the ortho-position, was isolated in reduced yield. Imidazolin-2-thiones with orthosubstituents were obtained as a pair of diastereoisomers but because the products of the subsequent oxidation, imidazolium salts $\mathbf{3 g}-\mathbf{4 i}$, do not exhibit atropoisomerism we did not separate the diastereoisomers. When the condensation was attempted with thioureas $\mathbf{1 k}, \mathbf{1 m}$, and $\mathbf{1 n}$, no imid- azolin-2-thiones were produced. Instead 2-[(2-tert-butylphenyl)amino]-1,2-diphenylethanone (5) was isolated (see Table 1 below). Attempts to achieve the cyclization in boiling hexanol in the presence of catalytic amounts of hydrochloric acid ${ }^{16}$ were unsuccessful. It should be noted that attempts to form $\mathrm{N}, \mathrm{N}$-bis(2-tert-butylphenyl)-4,5-dimethylimidazolin-2-thione failed, as reported by Bach et al. ${ }^{17}$ Interestingly, reaction of $N, N^{\prime}$-bis(3-pyridinyl)thiourea $\mathbf{1 1}$ with benzoin under similar conditions in acetic acid gave 2[(3-pyridyl)amino]-1,2-diphenylethanone (6) in excellent yield.

Oxidative desulfurization was performed using $\mathrm{H}_{2} \mathrm{O}_{2}$ in acetic acid. The initial products of oxidative desulfurization - the hydrogen sulfates ${ }^{8 b, c}$ were obtained as viscous syrups after careful removal of acetic acid. Ion exchange with $\mathrm{NaClO}_{4}$ gave perchlorates 3a-j effectively as analytically pure compounds. A mixture of two diastereoisomers was formed in the case of ortho-isopropyl-substituted imidazolium salt $\mathbf{3 j}$, as in the case of the 4,5-dimethyl analogue. ${ }^{17}$ We expect that the carbene generated from $3 \mathbf{j}$ will not exhibit atropoisomerism because rotation around the $\mathrm{N}-\mathrm{Ar}$ bond in the carbenes is not restricted. ${ }^{17}$ Therefore, no further attempts to separate the diastereoisomers were undertaken. Ion exchange with $\mathrm{BaCl}_{2}$ furnished imidazolium chlorides $\mathbf{4 a} \mathbf{a}-\mathbf{i}$. Unfortunately, desulfurization of $\mathbf{2} \mathbf{j}$ and ion exchange with $\mathrm{BaCl}_{2}$ did not give a crystalline product; therefore, no further attempts to purify this chloride salt were undertaken.

In conclusion, we have reported a convenient, largescale and cost-efficient synthesis of 4,5-diarylsubstituted imidazolium salts with electron-rich, and sterically demanding $\mathrm{N}$-aryl groups (Table 1 ). In this way, $\mathrm{N}, \mathrm{N}$-diarylimidazolium salts with ortho-methoxy, ortho-methyl, and ortho-isopropyl substituents can be obtained; whereas the synthesis of their 2-tert-butyl, 2,6-dimethyl, and 2,6-di-isopropyl analogues failed. 
3

SynOpen

C. Azap et al.

THIEME

OPEN
ACCESS

PSP

Table 1 Yields of the Prepared Compounds

Thiourea $1 \quad$ Yield Imidazolin-2-thione 2

(\%)

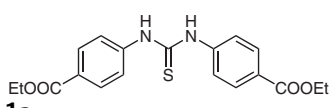

$1 \mathrm{a}$
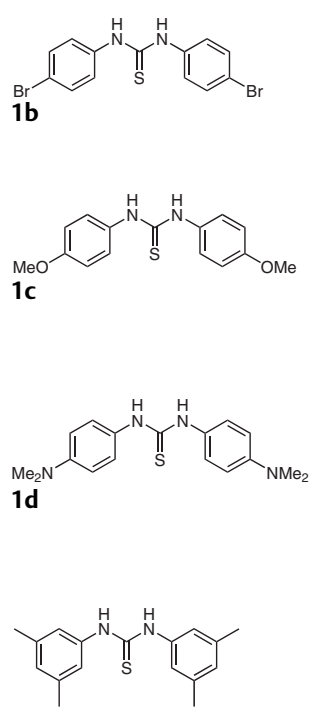

$1 \mathrm{e}$

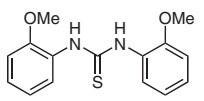

$1 \mathrm{~g}$
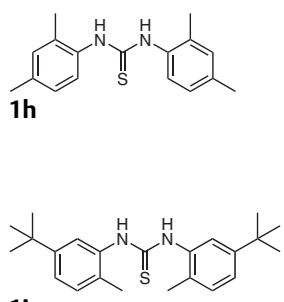

$1 \mathrm{i}$

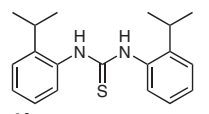

1j

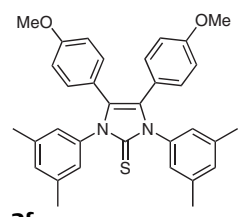

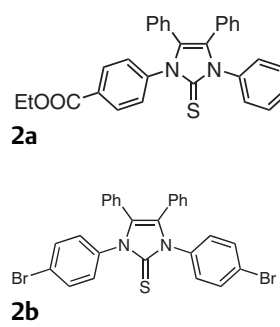

78

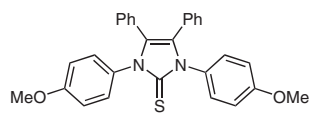

2c

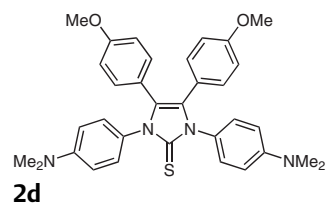

68

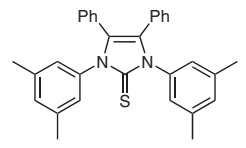

$2 e$

2f

61

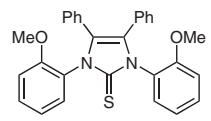

$2 g$

98

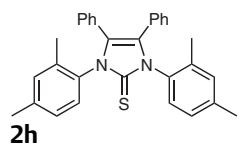

62

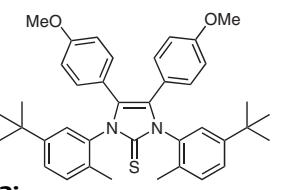

2i

56

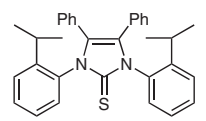

$2 \mathrm{j}$

86

88

61

63

31
Yield Imidazolium perchlorate $\mathbf{3}$

(\%)
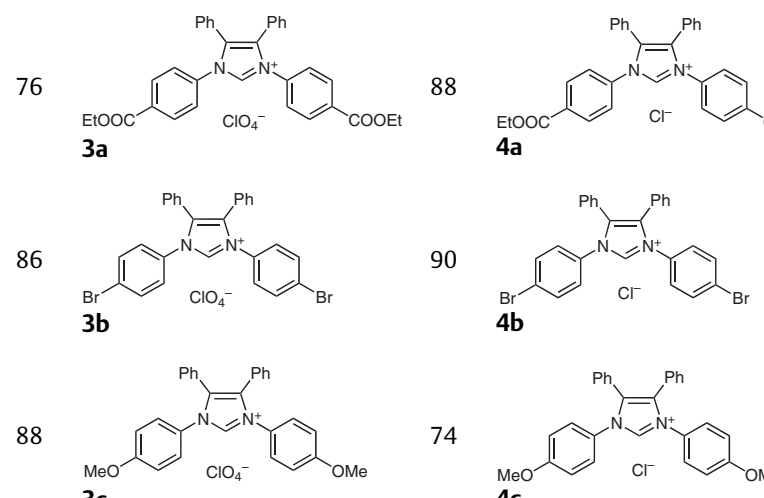

3c

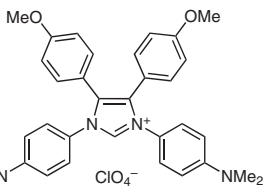

3d

$3 e$

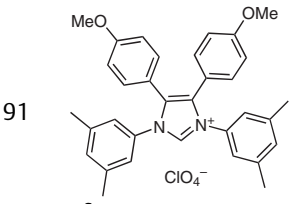

$3 f$

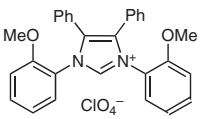

3g
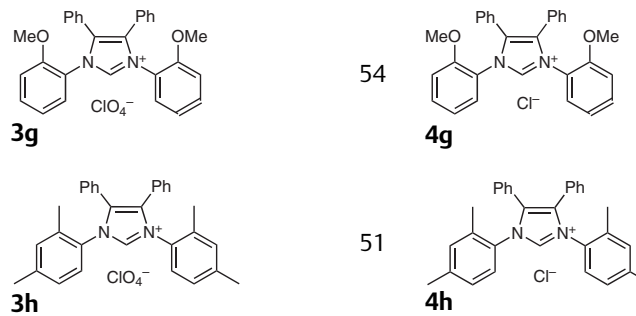

51

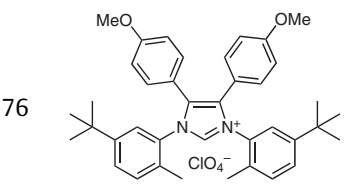

$3 \mathbf{i}$

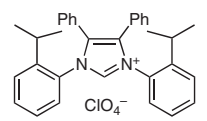

3j
90

74

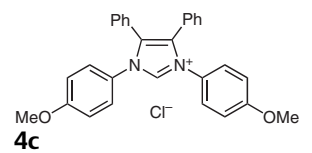

31

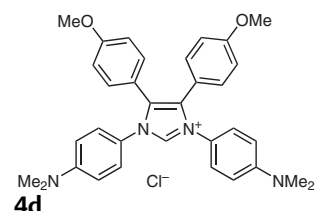

4d

64

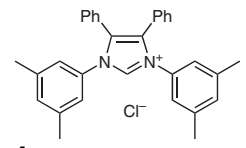

$4 \mathrm{e}$

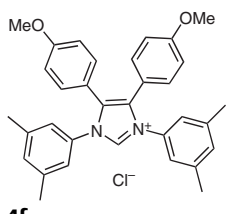

$4 f$

54

4g

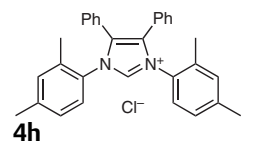

73

89

45

54

81

58

69

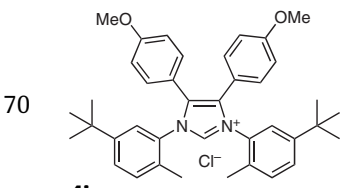

$4 \mathbf{i}$

$81-$

70

SynOpen 2020, 4, 1-11 
Table 1 (continued)

Yield Imidazolin-2-thione 2
$(\%)$

All starting materials and solvents were obtained from commercial suppliers and were used without further purification. 2-Methyl-5tert-butylaniline was prepared according to the reported procedure. ${ }^{18}$ Melting points of all synthesized compounds were determined in open capillaries and are uncorrected. NMR spectra were recorded with a Bruker Avance $400\left({ }^{1} \mathrm{H}: 400 \mathrm{MHz},{ }^{13} \mathrm{C}: 101 \mathrm{MHz}\right)$, Bruker Avance $600\left({ }^{1} \mathrm{H}: 601 \mathrm{MHz},{ }^{13} \mathrm{C}\right.$ : $\left.151 \mathrm{MHz}\right)$, or Jeol ECZ-600 R $\left({ }^{1} \mathrm{H}: 600\right.$ $\mathrm{MHz},{ }^{13} \mathrm{C}: 151 \mathrm{MHz}$ ) spectrometer; chemical shifts are given in $\delta \mathrm{ppm}$. IR spectra were recorded with a Bruker Tensor 27 spectrophotometer equipped with a 'GoldenGate' diamond ATR unit.

\section{General Procedures for the Synthesis of Thioureas 1}

General Procedure A: A stirred mixture of aniline $(1 \mathrm{~mol})$, sulfur $(2 \mathrm{~g})$, and potassium carbonate $(2 \mathrm{~g})$ in water $(700 \mathrm{~mL})$ was heated to $80{ }^{\circ} \mathrm{C}$, carbon disulfide $(0.6-1.0 \mathrm{~mol})$ was added dropwise over 60 min and the mixture was then heated to reflux for $6 \mathrm{~h}$. After cooling to ambient temperature, the precipitated product was filtered off, washed with $1 \mathrm{M}$ hydrochloric acid, then with water, sucked as dry as possible and the crude product was recrystallized from boiling ethanol.

General Procedure B: A mixture of 2-isopropylaniline (135.0 g, 1 mol), sulfur ( $1 \mathrm{~g})$, and potassium carbonate $(2 \mathrm{~g})$ in diglyme $(1 \mathrm{~L})$ was heated to $80^{\circ} \mathrm{C}$, then carbon disulfide ( $38 \mathrm{~g}, 0.5 \mathrm{~mol}$ ) was added over $1 \mathrm{~h}$. The mixture was then slowly heated to $180^{\circ} \mathrm{C}$ (bath temperature) and stirred at this temperature for $6 \mathrm{~h}$. The reaction mixture was then allowed to cool overnight to ambient temperature. The precipitate was filtered off with suction, washed with $1 \mathrm{M}$ hydrochloric acid, then with water, sucked as dry as possible, washed with hexane and dried in vacuum.

\section{General Procedure for the Synthesis of Imidazolin-2-thiones 2}

Benzoin/anisoin $(0.1 \mathrm{~mol})$ and $N, N^{\prime}$-diarylthiourea $(0.1 \mathrm{~mol})$ were heated to reflux in glacial acetic acid $(150 \mathrm{~mL})$ for $6-16 \mathrm{~h}$ and the mixture was then allowed to cool to ambient temperature. EtOH $(50 \mathrm{~mL})$ was added and the suspension was stirred in an ice bath to complete the precipitation. The precipitated product was filtered off with suction, washed with ethanol, then diethyl ether, and dried in vacuum.

\section{General Procedure for the Synthesis of Perchlorates 3}

Caution: Perchlorates are potentially explosive AND SHOULD BE TREated AS POTENTIALLY HAZARDOUS COMPOUNDS.

To a solution of imdiazolin-2-thione $\mathbf{2}(0.1 \mathrm{~mol})$ in glacial acetic acid (200 mL), 35\% aqueous $\mathrm{H}_{2} \mathrm{O}_{2}(34 \mathrm{~mL}, 0.4 \mathrm{~mol}$ ) was added dropwise, allowing the temperature to rise to $50{ }^{\circ} \mathrm{C}$, leading to a slightly turbid mixture that was subsequently stirred at ambient temperature for 4 h. All volatiles were removed by rotary evaporation (CAUTION: Do not evaporate to dryness. When preparing larger volumes, it is critical to ensure that any excess of hydrogen peroxide is destroyed before workup; otherwise, explosive decomposition may occur). The residue was dissolved in $\mathrm{MeOH}(200 \mathrm{~mL})$ and treated with a solution of $\mathrm{NaClO}_{4}(28.1$ $\mathrm{g}, 0.2 \mathrm{~mol})$ in a $2: 1(\mathrm{v} / \mathrm{v})$ mixture of methanol/water $(200 \mathrm{~mL})$. A white solid precipitated upon additional stirring in an ice bath. The precipitate was filtered off and washed with water, diethyl ether and dried at r.t. in vacuum.

\section{General Procedure for the Synthesis of Chlorides 4}

Hydrogen peroxide (35\%; $53 \mathrm{~mL}, 0.65 \mathrm{~mol}$ ) was added dropwise to a stirred suspension of imidazolin-2-thione $\mathbf{2}(0.2 \mathrm{~mol})$ in glacial acetic acid $(200 \mathrm{~mL})$. An exothermic reaction occurred with the reaction mixture reaching $60{ }^{\circ} \mathrm{C}$ at the end of the addition and the solution was stirred for a further $4 \mathrm{~h}$. All volatiles were removed by rotary evaporation (Caution: Do not evaporate to dryness. When preparing larger volumes, it is critical to ensure that any excess of hydrogen peroxide is destroyed before workup; otherwise, explosive decomposition may occur) and the residue was dissolved in $\mathrm{MeOH}(500 \mathrm{~mL})$. A solution of $\mathrm{BaCl}_{2}$ (97.7 g, $\left.0.4 \mathrm{~mol}\right)$ in water $(200 \mathrm{~mL})$ was added, and the suspension was filtered with suction through a G4 glass sinter covered with Celite ${ }^{\circledR}$. The filtrate was evaporated and the residue was extracted with dichloromethane $(3 \times 100 \mathrm{~mL})$. The combined organic layers were dried over $\mathrm{MgSO}_{4}$, filtered, and the filtrate evaporated. The residue was triturated with diethyl ether, the solid product was filtered with suction, washed with diethyl ether, and dried in vacuum.

\section{$\boldsymbol{N}, \boldsymbol{N}^{\prime}$-Bis(4-ethoxycarbonylphenyl)thiourea (1a) $)^{19}$}

According to GP B, the reaction of ethyl 4-aminobenzoate (82.6 g, 0.5 mol) and carbon disulfide $(45 \mathrm{~mL}, 0.75 \mathrm{~mol})$ and recrystallization from boiling water afforded 1a. An analytically pure sample was obtained by recrystallization from ethanol.

Yield: $47 \%$ (44.1 g); mp $164-165{ }^{\circ} \mathrm{C}\left(\mathrm{Lit}^{19} 165^{\circ} \mathrm{C}\right)$.

IR (ATR): 3284br, 1681s, 1591s, 1524s, 1507s, 1408s, 1328s, 1286s, 1229s, 1174s, 849s, 765s, 734s, 650s, 614s, 572s cm cm. $^{-1}$.

${ }^{1} \mathrm{H}$ NMR (400 MHz, DMSO- $d_{6}$ ): $\delta=10.33(\mathrm{~s}, 1 \mathrm{H}), 7.93(\mathrm{~d}, J=8.7 \mathrm{~Hz}$, $2 \mathrm{H}), 7.69(\mathrm{~d}, J=8.7 \mathrm{~Hz}, 2 \mathrm{H}), 4.30(\mathrm{q}, J=7.1 \mathrm{~Hz}, 2 \mathrm{H}), 1.32(\mathrm{t}, J=7.1 \mathrm{~Hz}$, $3 \mathrm{H})$.

${ }^{13} \mathrm{C}$ NMR (101 MHz, DMSO- $\left.d_{6}\right): \delta=179.09,165.23,143.71,129.66$, 125.04, 122.01, 60.44, 14.10.

Anal. Calcd for $\mathrm{C}_{19} \mathrm{H}_{20} \mathrm{~N}_{2} \mathrm{O}_{4} \mathrm{~S}$ : C, 61.27; H, 5.41; N, 7.52; S, 8.61. Found: C, 60.93; H, 5.38; N, 7.41; S, 8.72. 


\section{1,3-Bis(4-ethoxycarbonylphenyl)-4,5-diphenylimidazolin-2-thi-} one (2a)

Reaction of thiourea $1 \mathrm{a}(44.0 \mathrm{~g}, 0.12 \mathrm{~mol})$ and benzoin $(25.1 \mathrm{~g}, 0.12$ $\mathrm{mol})$ in acetic acid $(170 \mathrm{~mL})$ for $6 \mathrm{~h}$ afforded $2 \mathrm{a}$. An analytically pure sample was obtained by recrystallization from $\mathrm{CHCl}_{3} / \mathrm{EtOH}$.

Yield: $76 \%$ (49.1 g); $\mathrm{mp} 305-307^{\circ} \mathrm{C}$ (dec.).

IR (ATR): 1715s, 1606m, 1512m, 1336s, 1269s, 1172m, 1099s, 741s, 693s, $597 \mathrm{~s} \mathrm{~cm}^{-1}$.

${ }^{1} \mathrm{H} \mathrm{NMR}\left(601 \mathrm{MHz}, \mathrm{CDCl}_{3}\right): \delta=8.02-7.96(\mathrm{~m}, 4 \mathrm{H}), 7.42-7.35(\mathrm{~m}, 4 \mathrm{H})$, $7.11(\mathrm{t}, J=7.4 \mathrm{~Hz}, 2 \mathrm{H}), 7.05(\mathrm{t}, J=7.6 \mathrm{~Hz}, 4 \mathrm{H}), 6.92-6.87(\mathrm{~m}, 4 \mathrm{H})$, $4.29(\mathrm{q}, J=7.1 \mathrm{~Hz}, 4 \mathrm{H}), 1.30(\mathrm{t}, J=7.1 \mathrm{~Hz}, 6 \mathrm{H})$.

${ }^{13} \mathrm{C}$ NMR (151 MHz, $\left.\mathrm{CDCl}_{3}\right): \delta=165.78,165.69,140.60,130.54$, 130.33, 130.26, 129.06, 128.64, 128.51, 128.47, 127.50, 61.25, 14.30.

Anal. Calcd for $\mathrm{C}_{33} \mathrm{H}_{28} \mathrm{~N}_{2} \mathrm{O}_{4} \mathrm{~S}$ : C, 72.24; H, 5.14; N, 5.11; S, 5.84. Found: C, 71.86; H, 5.15; N, 5.02; S, 5.96.

\section{1,3-Bis(4-ethoxycarbonylphenyl)-4,5-diphenylimidazolium Per- chlorate (3a)}

Reaction of imidazolinthione $\mathbf{2 a}(13.72 \mathrm{~g}, 25 \mathrm{mmol})$ and $\mathrm{H}_{2} \mathrm{O}_{2}(8.8 \mathrm{~mL}$, $35 \%)$ in acetic acid $(90 \mathrm{~mL})$ afforded 3a. An analytically pure sample was obtained by recrystallization from $\mathrm{CHCl}_{3} / \mathrm{Et}_{2} \mathrm{O}$.

Yield: $88 \%$ (13.6 g); $\mathrm{mp} 336-338^{\circ} \mathrm{C}$ (dec.).

IR (ATR): 1715s, 1607w, 1542m, 1276s, 1086s, 1020m, 772s, 702s, $621 \mathrm{~s} \mathrm{~cm}^{-1}$

${ }^{1} \mathrm{H}$ NMR $\left(601 \mathrm{MHz}\right.$, DMSO- $\left.d_{6}\right): \delta=10.31(\mathrm{~s}, 1 \mathrm{H}), 8.14(\mathrm{~d}, J=8.5 \mathrm{~Hz}$, $4 \mathrm{H}), 7.70(\mathrm{~d}, J=8.5 \mathrm{~Hz}, 4 \mathrm{H}), 7.41(\mathrm{t}, J=7.3 \mathrm{~Hz}, 2 \mathrm{H}), 7.36(\mathrm{t}, J=7.4 \mathrm{~Hz}$, $4 \mathrm{H}), 7.26(\mathrm{~d}, J=7.2 \mathrm{~Hz}, 4 \mathrm{H}), 4.35(\mathrm{q}, J=7.1 \mathrm{~Hz}, 4 \mathrm{H}), 1.34(\mathrm{t}, J=7.1 \mathrm{~Hz}$, $6 \mathrm{H})$.

${ }^{13} \mathrm{C}$ NMR (151 MHz, DMSO- $d_{6}$ ): $\delta=165.05,138.22,137.58,132.10$, 132.06, 131.33, 131.04, 130.68, 129.35, 127.44, 125.07, 61.87, 14.54 .

Anal. Calcd for $\mathrm{C}_{33} \mathrm{H}_{29} \mathrm{ClN}_{2} \mathrm{O}_{8}$ : C, 64.24; $\mathrm{H}, 4.74 ; \mathrm{N}, 4.54$. Found: $\mathrm{C}$, $64.05 ; \mathrm{H}, 4.67 ; \mathrm{N}, 4.42$.

\section{1,3-Bis(4-ethoxycarbonylphenyl)-4,5-diphenylimidazolium Chlo- ride (4a)}

Reaction of imidazolinthione $\mathbf{2 a}$ (13.72 g, $25 \mathrm{mmol}$ ) and $\mathrm{H}_{2} \mathrm{O}_{2}(18 \mathrm{~mL}$, $35 \%)$ in acetic acid $(90 \mathrm{~mL})$ afforded $\mathbf{4 a}$ after crystallization from $\mathrm{CH}_{2} \mathrm{Cl}_{2} /$ EtOAc.

Yield: $68 \%$ (9.4 g); mp $277-278{ }^{\circ} \mathrm{C}$.

IR (ATR): 1702s, 1608w, 1560m, 1260s, 1175w, 1095s, 1025m, 869s, $771 \mathrm{~s}, 692 \mathrm{~s} \mathrm{~cm}^{-1}$.

${ }^{1} \mathrm{H}$ NMR (601 MHz, DMSO- $\left.d_{6}\right): \delta=10.39(\mathrm{~s}, 1 \mathrm{H}), 8.15-8.11(\mathrm{~m}, 4 \mathrm{H})$, $7.73(\mathrm{~d}, J=8.6 \mathrm{~Hz}, 4 \mathrm{H}), 7.42-7.39(\mathrm{~m}, 2 \mathrm{H}), 7.36(\mathrm{t}, J=7.4 \mathrm{~Hz}, 4 \mathrm{H})$, 7.30-7.26 (m, $4 \mathrm{H}), 4.35$ (q, J = 7.1 Hz, $4 \mathrm{H}), 1.34$ (t, J = 7.1 Hz, $6 \mathrm{H}$ ).

${ }^{13} \mathrm{C}$ NMR (151 MHz, DMSO- $\left.d_{6}\right): \delta=165.07,138.26,137.62,132.06$, 132.05, 131.36, 130.99, 130.65, 129.32, 127.50, 125.12, 61.86, 14.55.

Anal. Calcd for $\mathrm{C}_{33} \mathrm{H}_{29} \mathrm{ClN}_{2} \mathrm{O}_{4}$ : C, 71.67; H, 5.29; N, 5.07. Found: C, 71.28; H, 5.20; N, 4.86 .

\section{$\boldsymbol{N}, \boldsymbol{N}^{\prime}$-Bis(4-bromophenyl)thiourea (1b) $)^{20,21}$}

According to GP B, reaction of 4-bromoaniline $(86.0 \mathrm{~g}, 0.5 \mathrm{~mol})$ and carbon disulfide ( $45 \mathrm{~mL}, 0.75 \mathrm{~mol}$ ), afforded $\mathbf{1 b}$. An analytically pure sample was obtained by recrystallization from ethanol.

Yield: $85 \%$ (81.8 g); $\mathrm{mp} 188-189^{\circ} \mathrm{C}\left(\right.$ Lit. $\left.^{20} 188^{\circ} \mathrm{C}\right)$.

IR (ATR): 3206m, 3013m, 1588m, 1530s, 1482s, 1305s, 1068s, 1008s, $821 \mathrm{~s}, 717 \mathrm{~s} \mathrm{~cm}^{-1}$.
${ }^{1} \mathrm{H}$ NMR $\left(601 \mathrm{MHz}\right.$, DMSO- $\left.d_{6}\right): \delta=9.94(\mathrm{~s}, 2 \mathrm{H}), 7.54-7.51(\mathrm{~m}, 4 \mathrm{H})$, 7.49-7.45 (m, $4 \mathrm{H})$.

${ }^{13} \mathrm{C}$ NMR (151 MHz, DMSO- $\left.d_{6}\right): \delta=180.09,139.25,131.74,126.11$, 117.04 .

NMR spectroscopic data for $\mathbf{1 b}$ match those previously described. ${ }^{21}$

1,3-Bis(4-bromophenyl)-4,5-diphenylimidazolin-2-thione (2b)

Reaction of thiourea $\mathbf{1 b}(77.22 \mathrm{~g}, 0.2 \mathrm{~mol})$ and benzoin (42.42 g, 0.2 $\mathrm{mol})$ in acetic acid $(200 \mathrm{~mL})$ for $11 \mathrm{~h}$ afforded $\mathbf{2 b}$.

Yield: $86 \%$ (96.8 g); $\mathrm{mp} 319-320^{\circ} \mathrm{C}$.

IR (ATR): 2948w, 1487s, 1356s, 1069m, 1012m, 749s, 696s, 661 m cm1 .

${ }^{1} \mathrm{H}$ NMR $\left(601 \mathrm{MHz}\right.$, DMSO- $\left.d_{6}\right): \delta=7.63-7.59(\mathrm{~m}, 2 \mathrm{H}), 7.37-7.33(\mathrm{~m}$, $2 \mathrm{H}), 7.25-7.19(\mathrm{~m}, 3 \mathrm{H}), 7.16(\mathrm{dt}, J=3.9,2.3 \mathrm{~Hz}, 2 \mathrm{H})$.

${ }^{13} \mathrm{C}$ NMR (151 MHz, DMSO- $\left.d_{6}\right): \delta=165.29,136.55,132.24,131.75$, 131.10, 129.10, 128.72, 128.51, 128.07, 122.03.

Anal. Calcd for $\mathrm{C}_{27} \mathrm{H}_{18} \mathrm{Br}_{2} \mathrm{~N}_{2} \mathrm{~S}$ : C, 57.67; H, 3.23; N, 4.98; S, 5.70. Found: C, 57.38; H, 3.30; N, 4.79; S, 5.92 .

\section{1,3-Bis(4-bromophenyl)-4,5-diphenylimidazolium Perchlorate} (3b)

Reaction of imidazolinethione $\mathbf{2 b}(22.49 \mathrm{~g}, 40 \mathrm{mmol})$ and $\mathrm{H}_{2} \mathrm{O}_{2}(13.7$ $\mathrm{mL}, 35 \%)$ in acetic acid $(90 \mathrm{~mL})$ afforded product $\mathbf{3 b}$.

Yield: $90 \%$ (22.6 g); $\mathrm{mp} 319-320{ }^{\circ} \mathrm{C}$ (dec.).

IR (ATR): 1545m, 1485s, 1079s, 1012s, 1013s, 831m, 751s, 696s cm${ }^{-1}$. ${ }^{1} \mathrm{H}$ NMR $\left(601 \mathrm{MHz}\right.$, DMSO- $\left.d_{6}\right): \delta=10.18(\mathrm{~s}, 1 \mathrm{H}), 7.84-7.80(\mathrm{~m}, 4 \mathrm{H})$, 7.52-7.48 (m, 4 H), 7.43-7.39 (m, 2 H), 7.39-7.34 (m, 4 H), 7.28-7.25 (m, $4 \mathrm{H})$.

${ }^{13} \mathrm{C}$ NMR (151 MHz, DMSO- $d_{6}$ ): $\delta=138.03,133.30,133.27,132.00$, 131.36, 130.64, 129.32, 128.99, 125.12, 124.33.

Anal. Calcd for $\mathrm{C}_{27} \mathrm{H}_{19} \mathrm{Br}_{2} \mathrm{ClN}_{2} \mathrm{O}_{4}$ : $\mathrm{C}, 51.42 ; \mathrm{H}, 3.04 ; \mathrm{N}, 4.44$. Found: $\mathrm{C}$, 51.19; H, 3.09; N, 4.35 .

\section{1,3-Bis(4-bromophenyl)-4,5-diphenylimidazolium Chloride (4b)}

Reaction of imidazolinethione $\mathbf{2 b}(22.49 \mathrm{~g}, 40 \mathrm{mmol})$ and $\mathrm{H}_{2} \mathrm{O}_{2}(12$ $\mathrm{mL}, 35 \%)$ in acetic acid $(100 \mathrm{~mL})$ afforded product $\mathbf{4 b}$ after crystallization from $\mathrm{CH}_{2} \mathrm{Cl}_{2} /$ EtOAc.

Yield: $73 \%$ (16.5 g); $\mathrm{mp} 292-293{ }^{\circ} \mathrm{C}$ (dec.).

IR (ATR): 2970br, 1547s, 1483s, 1249m, 1017s, 830s, 750s, 695s cm-1. ${ }^{1} \mathrm{H} \mathrm{NMR}\left(601 \mathrm{MHz}, \mathrm{CDCl}_{3}\right): \delta=10.56(\mathrm{~s}, 1 \mathrm{H}), 7.58(\mathrm{~d}, J=8.7 \mathrm{~Hz}, 4 \mathrm{H})$, $7.49(\mathrm{~d}, J=8.7 \mathrm{~Hz}, 4 \mathrm{H}), 7.29(\mathrm{t}, J=7.5 \mathrm{~Hz}, 2 \mathrm{H}), 7.21(\mathrm{t}, J=7.9 \mathrm{~Hz}, 4 \mathrm{H})$, $7.11(\mathrm{dd}, J=8.2,1.0 \mathrm{~Hz}, 4 \mathrm{H})$.

${ }^{13} \mathrm{C}$ NMR $\left(151 \mathrm{MHz}, \mathrm{CDCl}_{3}\right): \delta=137.41,133.11,132.32,132.26$, $130.91,130.40,129.04,128.08,124.99,124.36$.

Anal. Calcd for $\mathrm{C}_{27} \mathrm{H}_{19} \mathrm{Br}_{2} \mathrm{ClN}_{2}$ : C, 57.22; H, 3.38; N, 4.94. Found: C, 56.84; H, 3.42; N, 4.85 .

$N, N^{\prime}$-Bis(4-methoxyphenyl)thiourea (1c) $)^{22-24}$

According to GP A, reaction of $p$-anisidine $(123.2 \mathrm{~g}, 1 \mathrm{~mol})$ and carbon disulfide (33 mL, $0.55 \mathrm{~mol}$ ), afforded 1c after recrystallization from EtOH.

Yield: $78 \%$ (224.9 g); $\mathrm{mp} 194-195{ }^{\circ} \mathrm{C}$ (dec.) (Lit. $188-189{ }^{\circ} \mathrm{C},{ }^{22}$ $\left.198^{\circ} \mathrm{C}^{23}\right)$.

IR (ATR): 3222br, 1612m, 1537s, 1507s, 1337s, 1284s, 1235s, 1032s, 837s, 725s, 671s, $581 \mathrm{~s} \mathrm{~cm}^{-1}$. 
${ }^{1} \mathrm{H}$ NMR $\left(600 \mathrm{MHz}\right.$, DMSO- $\left.d_{6}\right): \delta=9.37(\mathrm{~s}, 2 \mathrm{H}), 7.29(\mathrm{~d}, J=8.9 \mathrm{~Hz}$, $4 \mathrm{H}), 6.86(\mathrm{~d}, J=8.9 \mathrm{~Hz}, 4 \mathrm{H}), 3.71(\mathrm{~s}, 6 \mathrm{H})$.

${ }^{13} \mathrm{C}$ NMR (151 MHz, DMSO- $d_{6}$ ): $\delta=180.80,157.07,132.81,126.61$, 114.19, 55.78 .

NMR spectroscopic data of $\mathbf{1 c}$ match those previously described. ${ }^{24}$

1,3-Bis-(4-methoxyphenyl)-4,5-diphenylimidazolin-2-thione (2c)

Reaction of thiourea $1 \mathrm{c}(34.41 \mathrm{~g}, 0.12 \mathrm{~mol})$ and benzoin $(25.2 \mathrm{~g}, 0.12$ $\mathrm{mol})$ in acetic acid $(100 \mathrm{~mL})$ for $11 \mathrm{~h}$ and crystallization of the resultant product from EtOH afforded $2 \mathrm{c}$.

Yield: $88 \%$ ( $48.87 \mathrm{~g}$ ); $\mathrm{mp} 271-272{ }^{\circ} \mathrm{C}$.

IR (ATR): 1597m, 1511s, 1441s, 1340s, 1298s, 1241s, 1165s, 1028s, 836s, 784s, 696s, $576 \mathrm{~s} \mathrm{~cm}^{-1}$.

${ }^{1} \mathrm{H}$ NMR $\left(600 \mathrm{MHz}\right.$, DMSO- $\left.d_{6}\right): \delta=7.23(\mathrm{~d}, J=8.6 \mathrm{~Hz}, 4 \mathrm{H}), 7.13(\mathrm{dt}, J=$ $8.2,4.1 \mathrm{~Hz}, 6 \mathrm{H}), 7.09$ (d, J = 6.6 Hz, $4 \mathrm{H}), 6.88(\mathrm{~d}, J=8.6 \mathrm{~Hz}, 4 \mathrm{H}), 3.71$ (s, $6 \mathrm{H})$.

${ }^{13} \mathrm{C}$ NMR (151 MHz, DMSO- $\left.d_{6}\right): \delta=165.95,159.23,131.14,130.76$, $130.18,128.81,128.69,128.61,114.36,55.82$.

Anal. Calcd for $\mathrm{C}_{29} \mathrm{H}_{24} \mathrm{~N}_{2} \mathrm{O}_{2} \mathrm{~S}$ : C, 74.97; H, 5.21; N, 6.03; S, 6.90. Found: C, 74.69; H, 5.20; N, 5.95; S, 6.92.

\section{1,3-Bis(4-methoxyphenyl)-4,5-diphenylimidazolium Perchlorate (3c)}

Reaction of imidazolinethione $2 \mathbf{c}(10.0 \mathrm{~g}, 22 \mathrm{mmol})$ and $\mathrm{H}_{2} \mathrm{O}_{2}(7.8 \mathrm{~mL}$, $35 \%)$ in acetic acid $(100 \mathrm{~mL})$ afforded $3 \mathbf{c}$ after recrystallization from $\mathrm{MeOH}(1 \mathrm{~L})$.

Yield: $74 \%$ (8.45 g); $\mathrm{mp} 289-291{ }^{\circ} \mathrm{C}$.

IR (ATR): 1546m, 1508s, 1447m, 1253s, 1177m,1088s, 833s, 697s, $621 \mathrm{~s} \mathrm{~cm}^{-1}$.

${ }^{1} \mathrm{H}$ NMR $\left(600 \mathrm{MHz}\right.$, DMSO- $\left.d_{6}\right): \delta=9.97(\mathrm{~s}, 1 \mathrm{H}), 7.48-7.40(\mathrm{~m}, 4 \mathrm{H})$, 7.32 (dd, $J=11.1,3.9 \mathrm{~Hz}, 2 \mathrm{H}), 7.28(\mathrm{t}, J=7.5 \mathrm{~Hz}, 4 \mathrm{H}), 7.25-7.20(\mathrm{~m}$, $4 \mathrm{H}), 7.04$ (dd, $J=14.0,5.5 \mathrm{~Hz}, 4 \mathrm{H}), 3.75(\mathrm{~s}, 6 \mathrm{H})$.

${ }^{13} \mathrm{C}$ NMR (151 MHz, DMSO- $\left.d_{6}\right): \delta=160.88,137.83,132.27,131.46$, $130.37,129.13,128.44,126.82,125.76,115.28,56.18$.

Anal. Calcd for $\mathrm{C}_{29} \mathrm{H}_{25} \mathrm{ClN}_{2} \mathrm{O}_{6}$ : C, 65.35; H, 4.73; N, 5.26. Found: C, 64.64; H, 4.52; N, 4.95 .

\section{1,3-Bis(4-methoxyphenyl)-4,5-diphenylimidazolium Chloride $(4 c)^{8 e}$}

Reaction of imidazolinethione $2 \mathrm{c}(23.2 \mathrm{~g}, 50 \mathrm{mmol})$ and $\mathrm{H}_{2} \mathrm{O}_{2}(18 \mathrm{~mL}$, $35 \%)$ in acetic acid $(150 \mathrm{~mL})$ afforded $\mathbf{4 c}$. An analytical pure sample was obtained by recrystallization from $\mathrm{CH}_{2} \mathrm{Cl}_{2} / \mathrm{Et}_{2} \mathrm{O}$. Yield: $89 \%$ (20.83 g); mp $143-144{ }^{\circ} \mathrm{C}$.

IR (ATR): 3231w, 1550s, 1507s, 1449m, 1236s, 1177s, 1019s, 834s, $785 \mathrm{~s}, 699 \mathrm{~s} \mathrm{~cm}^{-1}$.

${ }^{1} \mathrm{H}$ NMR $\left(601 \mathrm{MHz}, \mathrm{CDCl}_{3}\right): \delta=10.37(\mathrm{~s}, 1 \mathrm{H}), 7.63(\mathrm{~d}, J=8.9 \mathrm{~Hz}, 4 \mathrm{H})$, $7.32(\mathrm{t}, J=7.5 \mathrm{~Hz}, 2 \mathrm{H}), 7.25(\mathrm{t}, J=7.7 \mathrm{~Hz}, 4 \mathrm{H}), 7.17(\mathrm{~d}, J=7.4 \mathrm{~Hz}, 4 \mathrm{H})$, $6.90(\mathrm{~d}, J=8.9 \mathrm{~Hz}, 4 \mathrm{H}), 3.79(\mathrm{~s}, 6 \mathrm{H})$.

${ }^{1} \mathrm{H}$ NMR data were in accordance with reported data. ${ }^{8 \mathrm{e}}$

${ }^{13} \mathrm{C}$ NMR (151 MHz, $\left.\mathrm{CDCl}_{3}\right): \delta=160.72,137.32,132.10,130.95$, $129.96,128.80,127.81,126.11,125.03,114.89,55.58$.

Anal. Calcd for $\mathrm{C}_{29} \mathrm{H}_{25} \mathrm{ClN}_{2} \mathrm{O}_{2}$ : C, 74.27; $\mathrm{H}, 5.37 ; \mathrm{N}, 5.97$. Found: C, 73.59; H, 5.40; N, 5.57 .

\section{$\boldsymbol{N}, \boldsymbol{N}^{\prime}$-Bis(4-dimethylaminophenyl)thiourea (1d) $)^{22}$}

According to GP A, reaction of $N, N$-dimethyl-p-phenylenediamine $(50.0 \mathrm{~g}, 0.37 \mathrm{~mol})$ and carbon disulfide $(12 \mathrm{~mL}, 0.2 \mathrm{~mol})$, followed by recrystallization from EtOH afforded 1d.

Yield: $72 \%$ (41.9 g); $\mathrm{mp} 189-190^{\circ} \mathrm{C}$ (dec.) (Lit. $22185-186{ }^{\circ} \mathrm{C}$ ). IR (ATR): 3300-3100br, 2884m, 1611s, 1519s, 1367s, 1231s, 1174s, 945s, 818s, 721s, 673s cm-1.

${ }^{1} \mathrm{H}$ NMR $\left(600 \mathrm{MHz}\right.$, DMSO- $\left.d_{6}\right): \delta=9.08(\mathrm{~s}, 2 \mathrm{H}), 7.16(\mathrm{~d}, J=8.9 \mathrm{~Hz}$, $4 \mathrm{H}), 6.65(\mathrm{~d}, J=9.0 \mathrm{~Hz}, 4 \mathrm{H}), 2.84(\mathrm{~s}, 12 \mathrm{H})$.

${ }^{13} \mathrm{C}$ NMR (151 MHz, DMSO- $\left.d_{6}\right): \delta=180.51,148.67,129.09,126.39$, 112.81, 40.93 .

Anal. Calcd for $\mathrm{C}_{17} \mathrm{H}_{22} \mathrm{~N}_{4} \mathrm{~S}$ : C, 64.93; H, 7.05; N, 17.82; S, 10.20. Found: C, 64.70; H, 7.02; N, 17.71; S, 10.31 .

\section{1,3-Bis(4-dimethylaminophenyl)-4,5-di(4-methoxyphenyl)imid- azolin-2-thione (2d)}

Reaction of thiourea 1d $(31.45 \mathrm{~g}, 0.1 \mathrm{~mol})$ and anisoin $(27.2 \mathrm{~g}, 0.1$ $\mathrm{mol}$ ) in acetic acid ( $100 \mathrm{~mL}$ ) for $7 \mathrm{~h}$ afforded $\mathbf{2 d}$ after recrystallization from EtOH. An analytically pure sample was obtained by recrystallization from $\mathrm{CHCl}_{3} / \mathrm{EtOH}$.

Yield: $61 \%$ (33.6 g); $\mathrm{mp} 250-251{ }^{\circ} \mathrm{C}$ (dec.).

IR (ATR): 1610s, 1516s, 1342s, 1247s, 1172s, 1026s, 803s, 757s cm-1. ${ }^{1} \mathrm{H}$ NMR $\left(601 \mathrm{MHz}, \mathrm{CDCl}_{3}\right): \delta=7.17(\mathrm{~d}, J=8.2 \mathrm{~Hz}, 4 \mathrm{H}), 6.91(\mathrm{~d}, J=$ $8.4 \mathrm{~Hz}, 4 \mathrm{H}), 6.66$ (d, J = 8.4 Hz, $4 \mathrm{H}), 6.64(\mathrm{~d}, J=8.4 \mathrm{~Hz}, 4 \mathrm{H}), 3.71$ (s, $6 \mathrm{H}), 2.95(\mathrm{~s}, 12 \mathrm{H})$.

${ }^{13} \mathrm{C}$ NMR $\left(151 \mathrm{MHz}, \mathrm{CDCl}_{3}\right): \delta=165.44,158.95,149.83,131.77$, $129.45,128.03,126.22,121.11,113.57,112.16,55.06,40.48$.

Anal. Calcd for $\mathrm{C}_{33} \mathrm{H}_{34} \mathrm{~N}_{4} \mathrm{O}_{2} \mathrm{~S}$ : C, 71.97; H, 6.22; N, 10.17; S, 5.82. Found: C, 71.64; H, 6.11; N, 10.02; S, 5.39.

\section{1,3-Bis(4-dimethylaminophenyl)-4,5-di(4-methoxyphenyl)imid-} azolium Perchlorate (3d)

Reaction of imidazolinethione $2 \mathbf{d}(10.4 \mathrm{~g}, 20 \mathrm{mmol})$ and $\mathrm{H}_{2} \mathrm{O}_{2}(6 \mathrm{~mL}$, $35 \%)$ in acetic acid $(90 \mathrm{~mL})$ afforded, after crystallization from methanol, product 3d. An analytically pure sample was obtained by recrystallization from $\mathrm{CHCl}_{3} / \mathrm{MeOH}$.

Yield: $31 \%$ (3.4 g); mp $174-175^{\circ} \mathrm{C}$.

IR (ATR): 1612m, 1518s, 1244s, 1180m, 1089s, 818s, 620s cm-1.

${ }^{1} \mathrm{H}$ NMR $\left(600 \mathrm{MHz}\right.$, DMSO- $\left.d_{6}\right): \delta=9.73(\mathrm{~s}, 1 \mathrm{H}), 7.24(\mathrm{~d}, J=8.6 \mathrm{~Hz}$, $4 \mathrm{H}), 7.13$ (d, $J=8.4 \mathrm{~Hz}, 4 \mathrm{H}), 6.84(\mathrm{~d}, J=8.5 \mathrm{~Hz}, 4 \mathrm{H}), 6.71$ (d, $J=$ $8.8 \mathrm{~Hz}, 4 \mathrm{H}), 3.67$ (s, $6 \mathrm{H}), 2.90(\mathrm{~s}, 12 \mathrm{H})$.

${ }^{13} \mathrm{C}$ NMR (151 MHz, DMSO- $\left.d_{6}\right): \delta=160.43,151.36,136.90,132.88$, 131.92, 127.53, 122.40, 118.13, 114.59, 112.27, 55.67, 40.34.

Anal. Calcd for $\mathrm{C}_{33} \mathrm{H}_{35} \mathrm{ClN}_{4} \mathrm{O}_{6}$ : C, 64.02; H, 5.70; N, 9.05. Found: C, 63.62; H, 5.32; N, 8.91.

\section{1,3-Bis(4-dimethylaminophenyl)-4,5-di(4-methoxyphenyl)imid-} azolium Chloride (4d)

Reaction of imidazolinethione $2 \mathbf{d}(12.21 \mathrm{~g}, 22 \mathrm{mmol})$ and $\mathrm{H}_{2} \mathrm{O}_{2}(8 \mathrm{~mL}$, $35 \%)$ in acetic acid $(80 \mathrm{~mL})$ afforded $\mathbf{4 d}$.

Yield: $45 \%$ (5.53 g); $\mathrm{mp} 110-112{ }^{\circ} \mathrm{C}$.

IR (ATR): 1715m, 1518s, 1445m, 1228s, 1178s, 1025m, 821s, 605s $\mathrm{Cm}^{-1}$.

${ }^{1} \mathrm{H}$ NMR $\left(601 \mathrm{MHz}, \mathrm{CDCl}_{3}\right): \delta=8.52(\mathrm{~s}, 1 \mathrm{H}), 7.32(\mathrm{~d}, J=9.0 \mathrm{~Hz}, 1 \mathrm{H})$, $7.12(\mathrm{~d}, J=8.8 \mathrm{~Hz}, 1 \mathrm{H}), 6.69(\mathrm{~d}, J=8.8 \mathrm{~Hz}, 1 \mathrm{H}), 6.59(\mathrm{~d}, J=9.0 \mathrm{~Hz}$, $1 \mathrm{H}), 3.70$ (s, $6 \mathrm{H}), 2.93(\mathrm{~s}, 12 \mathrm{H})$. 
${ }^{13} \mathrm{C}$ NMR (151 MHz, $\left.\mathrm{CDCl}_{3}\right): \delta=160.26,150.99,134.22,132.55$, 132.41, 127.19, 122.05, 117.56, 114.05, 112.10, 55.17, 40.24.

Anal. Calcd for $\mathrm{C}_{33} \mathrm{H}_{35} \mathrm{ClN}_{4} \mathrm{O}_{2}$ : C, 71.40; H, 6.36; N, 10.09. Found: C, $71.01 ; \mathrm{H}, 6.34 ; \mathrm{N}, 9.87$.

\section{$\boldsymbol{N}, \boldsymbol{N}^{\prime}$-Bis(3,5-dimethylphenyl)thiourea (1e) $)^{25}$}

According to GP A, reaction of 3,5-dimethylaniline (121.2 g, $1 \mathrm{~mol}$ ) and carbon disulfide $(42 \mathrm{~g}, 0.55 \mathrm{~mol})$, after recrystallization from EtOH (800 $\mathrm{mL})$ afforded 1e.

Yield: $68 \%$ (96.1 g); $\mathrm{mp} 150-151{ }^{\circ} \mathrm{C}$.

IR (ATR): 3347s, 3200-2900br, 1607s, 1536s, 1508s, 1303s, 1272s, $1228 \mathrm{~s}, 854 \mathrm{~s}, 700 \mathrm{~s}, 652 \mathrm{~s} \mathrm{~cm}^{-1}$.

${ }^{1} \mathrm{H}$ NMR (600 MHz, DMSO- $d_{6}$ ): $\delta=9.49$ (s, $2 \mathrm{H}$ ), $7.02(\mathrm{~s}, 4 \mathrm{H}), 6.73(\mathrm{~s}$, $2 \mathrm{H}), 2.21(\mathrm{~s}, 12 \mathrm{H})$.

${ }^{13} \mathrm{C}$ NMR (151 MHz, DMSO- $\left.d_{6}\right): \delta=179.98,139.74,137.96,126.58$, 122.07, 21.48.

NMR data were in accordance with those described..$^{25}$

\section{1,3-Bis(3,5-dimethylphenyl)-4,5-diphenylimidazolin-2-thione (2e)}

Reaction of thiourea 1e (67.5 g, $0.25 \mathrm{~mol})$ and benzoin $(53.0 \mathrm{~g}, 0.25$ $\mathrm{mol})$ in acetic acid $(100 \mathrm{~mL})$ for $10 \mathrm{~h}$ afforded $\mathbf{2 e}$.

Yield: $87 \%$ (100.2 g); $\mathrm{mp} 239-240{ }^{\circ} \mathrm{C}$.

IR (ATR): 1594m, 1328s, 1258w, 723s, 689s, 610s, 581s cm ${ }^{-1}$.

${ }^{1} \mathrm{H}$ NMR (600 MHz, DMSO- $\left.d_{6}\right): \delta=7.24-7.01(\mathrm{~m}, 10 \mathrm{H}), 6.92(\mathrm{~s}, 6 \mathrm{H})$, $2.17(\mathrm{~s}, 12 \mathrm{H})$.

${ }^{13} \mathrm{C}$ NMR $\left(151 \mathrm{MHz}, \mathrm{DMSO}-d_{6}\right): \delta=165.50,138.29,137.31,131.08$, $130.24,128.81,128.64,128.58,128.53,127.20,21.18$.

Anal. Calcd for $\mathrm{C}_{31} \mathrm{H}_{28} \mathrm{~N}_{2} \mathrm{~S}$ : C, 80.83; H, 6.13; N, 6.08; S, 6.96. Found: C, 79.95; H, 6.11; N, 5.95; S, 7.32.

\section{1,3-Bis(3,5-dimethylphenyl)-4,5-diphenylimidazolium Perchlo- rate (3e)}

Reaction of imidazolinethione $2 \mathbf{e}(37.1 \mathrm{~g}, 80 \mathrm{mmol})$ and $\mathrm{H}_{2} \mathrm{O}_{2}(28 \mathrm{~mL}$, $35 \%)$ in acetic acid $(150 \mathrm{~mL})$ afforded $3 \mathbf{3}$. An analytically pure sample was obtained by recrystallization from $\mathrm{CHCl}_{3} / \mathrm{Et}_{2} \mathrm{O}$.

Yield: $64 \%$ (27.1 g); $\mathrm{mp} 276-277^{\circ} \mathrm{C}$.

IR (ATR): $1542 \mathrm{~m}, 1083 \mathrm{~s}, 864 \mathrm{~m}, 772 \mathrm{~m}, 697 \mathrm{~s}, 621 \mathrm{~s} \mathrm{~cm}^{-1}$.

${ }^{1} \mathrm{H}$ NMR (600 MHz, DMSO- $\left.d_{6}\right): \delta=10.00(\mathrm{~s}, 1 \mathrm{H}), 7.36-7.32(\mathrm{~m}, 2 \mathrm{H})$, $7.30(\mathrm{t}, J=7.4 \mathrm{~Hz}, 4 \mathrm{H}), 7.22(\mathrm{~d}, J=7.3 \mathrm{~Hz}, 4 \mathrm{H}), 7.16(\mathrm{~s}, 2 \mathrm{H}), 7.12(\mathrm{~s}$, $4 \mathrm{H}), 2.23(\mathrm{~s}, 12 \mathrm{H})$.

${ }^{13} \mathrm{C}$ NMR (151 MHz, DMSO- $d_{6}$ ): $\delta=139.68,137.65,133.99,132.19$, $132.04,131.42,130.49,129.18,125.61,124.42,21.18$.

Anal. Calcd for $\mathrm{C}_{31} \mathrm{H}_{29} \mathrm{ClN}_{2} \mathrm{O}_{4}$ : C, 70.38; H, 5.53; N, 5.30. Found: C, $69.91 ; \mathrm{H}, 5.51 ; \mathrm{N}, 5.01$.

\section{1,3-Bis(3,5-dimethylphenyl)-4,5-diphenylimidazolium Chloride (4e)}

Reaction of imidazolinthione $2 \mathbf{e}(6.9 \mathrm{~g}, 15 \mathrm{mmol})$ and $\mathrm{H}_{2} \mathrm{O}_{2}(5.3 \mathrm{~mL}$, $35 \%)$ in acetic acid (50 $\mathrm{mL}$ ) afforded $4 \mathbf{e}$.

Yield: $54 \%$ (3.77 g); $\mathrm{mp} 222-224{ }^{\circ} \mathrm{C}$ (dec.).

IR (ATR): 1719m, 1540m, $1256 \mathrm{~m}, 1022 \mathrm{~m}, 859 \mathrm{~m}, 690 \mathrm{~s} \mathrm{~cm}^{-1}$.

${ }^{1} \mathrm{H}$ NMR NMR (400 MHz, $\left.\mathrm{CDCl}_{3}\right): \delta=10.35(\mathrm{~s}, 1 \mathrm{H}), 7.35-7.30(\mathrm{~m}, 2 \mathrm{H})$, $7.27(\mathrm{~d}, J=2.5 \mathrm{~Hz}, 2 \mathrm{H}), 7.25-7.22(\mathrm{~m}, 6 \mathrm{H}), 7.19-7.14(\mathrm{~m}, 4 \mathrm{H}), 7.04(\mathrm{~s}$, $2 \mathrm{H}), 2.28$ (s, $12 \mathrm{H})$.
${ }^{13} \mathrm{C}$ NMR $\left(101 \mathrm{MHz}, \mathrm{CDCl}_{3}\right): \delta=139.80,137.06,133.20,132.00$, 131.93, 130.90, 129.90, 128.70, 125.12, 123.93, 21.14.

Anal. Calcd for $\mathrm{C}_{31} \mathrm{H}_{29} \mathrm{ClN}_{2}$ : C, 80.07; $\mathrm{H}, 6.29 ; \mathrm{N}, 6.02$. Found: C, 79.52; H, 6.22; N, 5.85 .

1,3-Bis(3,5-dimethylphenyl)-4,5-di(4-methoxyphenyl)imidazolin2-thione (2f)

Reaction of thiourea 1 e $(96.0 \mathrm{~g}, 0.34 \mathrm{~mol})$ and anisoin $(91.0 \mathrm{~g}, 0.34$ $\mathrm{mol})$ in acetic acid $(400 \mathrm{~mL})$ for $12 \mathrm{~h}$, after recrystallization from EtOH (1 L) afforded $\mathbf{2 f}$.

Yield: 91\% (161.2 g); $\mathrm{mp} 267-268^{\circ} \mathrm{C}$ (dec.).

IR (ATR): 1605m, 1505s, 1364s, 1327s, 1293s, 1246s, 1174s, 827s, $755 \mathrm{~s} \mathrm{~cm}^{-1}$.

${ }^{1} \mathrm{H}$ NMR $\left(400 \mathrm{MHz}, \mathrm{CDCl}_{3}\right): \delta=6.97-6.93(\mathrm{~m}, 6 \mathrm{H}), 6.92-6.87(\mathrm{~m}, 4 \mathrm{H})$, 6.65-6.60 (m, $4 \mathrm{H}), 3.69(\mathrm{~s}, 6 \mathrm{H}), 2.27(\mathrm{~s}, 12 \mathrm{H})$.

${ }^{13} \mathrm{C}$ NMR $\left(101 \mathrm{MHz}, \mathrm{CDCl}_{3}\right): \delta=164.71,159.14,138.47,137.00$, 131.72, 130.37, 128.04, 126.74, 120.70, 113.57, 55.08, 21.23.

Anal. Calcd for $\mathrm{C}_{33} \mathrm{H}_{32} \mathrm{~N}_{2} \mathrm{O}_{2} \mathrm{~S}$ : C, 76.12; H, 6.19; N, 5.38; S, 6.16. Found: C, 75.89; H, 6.20; N, 5.19; S, 6.49.

1,3-Bis(3,5-dimethylphenyl)-4,5-di(4-methoxyphenyl)imidazolium Perchlorate (3f)

Reaction of imidazolinethione $2 \mathbf{f}(52.0 \mathrm{~g}, 0.1 \mathrm{~mol})$ and $\mathrm{H}_{2} \mathrm{O}_{2}(34 \mathrm{~mL}$, $35 \%)$ in acetic acid (150 $\mathrm{mL}$ ) afforded $\mathbf{3 f}$.

Yield: $89 \%$ (52.3 g); $\mathrm{mp} 274-275{ }^{\circ} \mathrm{C}$ (dec.).

IR (ATR): $1614 \mathrm{~m}, 1506 \mathrm{~m}, 1473 \mathrm{~m}, 1255 \mathrm{~s}, 1082 \mathrm{~s}, 841 \mathrm{~s}, 620 \mathrm{~s} \mathrm{~cm}^{-1}$.

${ }^{1} \mathrm{H}$ NMR $\left(400 \mathrm{MHz}, \mathrm{CDCl}_{3}\right): \delta=9.70(\mathrm{~s}, 1 \mathrm{H}), 7.22(\mathrm{~s}, 4 \mathrm{H}), 7.09(\mathrm{t}, J=$ $8.4 \mathrm{~Hz}, 4 \mathrm{H}), 7.02(\mathrm{~s}, 2 \mathrm{H}), 6.72(\mathrm{~d}, J=8.4 \mathrm{~Hz}, 4 \mathrm{H}), 3.72(\mathrm{~s}, 6 \mathrm{H}), 2.27(\mathrm{~s}$, $12 \mathrm{H})$.

${ }^{13} \mathrm{C}$ NMR (151 MHz, DMSO- $\left.d_{6}\right): \delta=160.63,139.62,137.09,134.17$, 132.89, 132.12, 131.84, 124.52, 117.63, 114.63, 55.71, 21.20.

Anal. Calcd for $\mathrm{C}_{33} \mathrm{H}_{33} \mathrm{ClN}_{2} \mathrm{O}_{6}$ : C, 67.28; H, 5.65; N, 4.76. Found: C, $66.57 ; \mathrm{H}, 5.61 ; \mathrm{N}, 4.65$.

1,3-Bis(3,5-dimethylphenyl)-4,5-di(4-methoxyphenyl)imidazolium Chloride (4f)

Reaction of imidazolinethione $\mathbf{2 f}(104.0 \mathrm{~g}, 0.2 \mathrm{~mol})$ and $\mathrm{H}_{2} \mathrm{O}_{2}(53 \mathrm{~mL}$, $35 \%)$ in acetic acid (200 $\mathrm{mL})$ afforded $\mathbf{4 f}$.

Yield: $81 \%$ (84.5 g); $\mathrm{mp} 216-217^{\circ} \mathrm{C}$ (dec.).

IR (ATR): 1543m, 1249s, 1176s, 1023s, 833s, 695s, 584s cm-1.

${ }^{1} \mathrm{H}$ NMR $\left(600 \mathrm{MHz}\right.$, DMSO-d $\left.d_{6}\right): \delta=9.94(\mathrm{~s}, 1 \mathrm{H}), 7.18-7.13(\mathrm{~m}, 10 \mathrm{H})$, $6.84(\mathrm{~d}, J=7.9 \mathrm{~Hz}, 4 \mathrm{H}), 3.66(\mathrm{~d}, J=0.7 \mathrm{~Hz}, 6 \mathrm{H}), 2.23(\mathrm{~s}, 12 \mathrm{H})$.

${ }^{13} \mathrm{C}$ NMR (151 MHz, DMSO- $\left.d_{6}\right): \delta=160.65,139.57,137.14,134.19$, 132.93, 132.08, 131.85, 124.55, 117.68, 114.62, 55.74, 21.18.

Anal. Calcd for $\mathrm{C}_{33} \mathrm{H}_{33} \mathrm{ClN}_{2} \mathrm{O}_{2}$ : C, 75.48; $\mathrm{H}, 6.33 ; \mathrm{Cl}, \mathrm{N}, 5.34$. Found: $\mathrm{C}$, 74.65; H, 6.24; N, 5.06 .

\section{$N, N^{\prime}$-Bis(2-methoxyphenyl)thiourea (1g) $)^{26}$}

According to GP A, reaction of $o$-anisidine $(61.53 \mathrm{~g}, 0.5 \mathrm{~mol})$ and carbon disulfide ( $22.8 \mathrm{~g}, 0.3 \mathrm{~mol}$ ), after recrystallization from EtOH (200 $\mathrm{mL}$ ) afforded $\mathbf{1 g}$.

Yield: $56 \%$ (39.4 g); $\mathrm{mp} 136-137^{\circ} \mathrm{C}$ ( Lit. $^{26} 132-134{ }^{\circ} \mathrm{C}$ ).

IR (ATR): 3200-3100 br, 2958 br, 1509s, 1490s, 1455s, 1313s, 1258s, 1231s, 1163s, 1112s, 1024s, 778s, 754s, 645s cm-1. 
${ }^{1} \mathrm{H}$ NMR $\left(600 \mathrm{MHz}\right.$, DMSO- $\left.d_{6}\right): \delta=9.33(\mathrm{~s}, 1 \mathrm{H}), 7.93$ (dd, $J=7.9$, $1.0 \mathrm{~Hz}, 1 \mathrm{H}), 7.14-7.09$ (m, $1 \mathrm{H}), 7.03$ (dd, $J=8.2,1.1 \mathrm{~Hz}, 1 \mathrm{H}), 6.92-$ $6.87(\mathrm{~m}, 1 \mathrm{H}), 3.79(\mathrm{~s}, 3 \mathrm{H})$.

${ }^{13} \mathrm{C}$ NMR $\left(151 \mathrm{MHz}\right.$, DMSO- $\left.d_{6}\right): \delta=180.00,152.26,128.15,126.31$, 120.34, 111.96, 56.23.

Anal. Calcd for $\mathrm{C}_{15} \mathrm{H}_{16} \mathrm{~N}_{2} \mathrm{O}_{2} \mathrm{~S}$ : C, 62.48; H, 5.59; N, 9.71; S, 11.12. Found: C, 62.41; H, 5.60; N, 9.65; S, 11.10.

\section{1,3-Bis(2-methoxyphenyl)-4,5-diphenylimidazolin-2-thione (2g)}

Reaction of thiourea $1 \mathrm{~g}(39.4 \mathrm{~g}, 0.14 \mathrm{~mol})$ and benzoin $(28.8 \mathrm{~g}, 0.14$ $\mathrm{mol}$ ) in acetic acid $(70 \mathrm{~mL}$ ) for $6 \mathrm{~h}$ afforded $2 \mathrm{~g}$. An analytically pure sample was obtained by recrystallization from $\mathrm{CHCl}_{3}$.

Yield: $61 \%$ (38.5 g); $\mathrm{mp} 242-243{ }^{\circ} \mathrm{C}$.

IR (ATR): 1502m, 1377m, 1346s, 1253m, 1019m, 750s, 700s, 570s cm-1. ${ }^{1} \mathrm{H} \mathrm{NMR}\left(601 \mathrm{MHz}, \mathrm{CDCl}_{3}\right): \delta=7.59(\mathrm{~d}, J=7.6 \mathrm{~Hz}, 2 \mathrm{H}), 7.32(\mathrm{t}, J=$ $7.7 \mathrm{~Hz}, 2 \mathrm{H}), 7.14-7.10(\mathrm{~m}, 2 \mathrm{H}), 7.06(\mathrm{dd}, J=15.0,7.6 \mathrm{~Hz}, 6 \mathrm{H}), 7.02$ (d, $J=7.4 \mathrm{~Hz}, 4 \mathrm{H}), 6.83(\mathrm{~d}, J=8.3 \mathrm{~Hz}, 2 \mathrm{H}), 3.53(\mathrm{~s}, 6 \mathrm{H})$.

${ }^{13} \mathrm{C}$ NMR $\left(151 \mathrm{MHz}, \mathrm{CDCl}_{3}\right): \delta=154.73,131.27,130.44,129.91$, $128.77,128.71,127.89,127.74,125.95,120.66,112.35,55.54$.

Anal. Calcd for $\mathrm{C}_{29} \mathrm{H}_{24} \mathrm{~N}_{2} \mathrm{O}_{2} \mathrm{~S}$ : C, 74.97; H, 5.21; N, 6.03; S, 6.90. Found: C, 74.37; H, 5.20; N, 5.89; S, 6.91 .

\section{1,3-Bi-(2-methoxyphenyl)-4,5-diphenylimidazolium Perchlorate (3g)}

Reaction of imidazolinethione $\mathbf{2 g}(23.1 \mathrm{~g}, 50 \mathrm{mmol})$ and $\mathrm{H}_{2} \mathrm{O}_{2}(18 \mathrm{~mL}$, $35 \%)$ in acetic acid (50 $\mathrm{mL}$ ) afforded $\mathbf{3 g}$.

Yield: $54 \%$ (14.4 g); $\mathrm{mp} 197-200{ }^{\circ} \mathrm{C}$.

IR (ATR): $1548 \mathrm{~m}, 1442 \mathrm{~m}, 1259 \mathrm{~m}, 1083 \mathrm{~s}, 761 \mathrm{~s}, 695 \mathrm{~s}, 623 \mathrm{~s} \mathrm{~cm}^{-1}$.

${ }^{1} \mathrm{H}$ NMR $\left(600 \mathrm{MHz}\right.$, DMSO- $\left.d_{6}\right): \delta=9.95(\mathrm{~s}, 1 \mathrm{H}), 7.56(\mathrm{dd}, J=7.8$, $1.4 \mathrm{~Hz}, 2 \mathrm{H}$ ), 7.50-7.43 (m, $2 \mathrm{H}), 7.29-7.23(\mathrm{~m}, 2 \mathrm{H}), 7.20$ (t, J = 7.6 Hz, $4 \mathrm{H}$ ), 7.12 (dd, $J=12.1,4.6 \mathrm{~Hz}, 2 \mathrm{H}$ ), 7.11-7.07 (m, $4 \mathrm{H}$ ), 7.04 (dd, $J=$ $11.3,4.0 \mathrm{~Hz}, 2 \mathrm{H}), 3.56$ (s, $6 \mathrm{H})$.

${ }^{13} \mathrm{C}$ NMR $\left(151 \mathrm{MHz}\right.$, DMSO- $\left.d_{6}\right): \delta=153.89,139.22,133.12,132.37$, 130.56, 130.36, 129.08, 128.98, 125.65, 122.30, 121.50, 113.64, 56.58. Anal. Calcd for $\mathrm{C}_{29} \mathrm{H}_{25} \mathrm{ClN}_{2} \mathrm{O}_{6}$ : C, 65.35; H, 4.73; N, 5.26. Found: C, $64.84 ; \mathrm{H}, 4.71 ; \mathrm{N}, 5.23$.

1,3-Bis(2-methoxyphenyl)-4,5-diphenylimidazolium Chloride (4g) Reaction of imidazolinethione $2 \mathrm{~g}(18.57 \mathrm{~g}, 40 \mathrm{mmol})$ and $\mathrm{H}_{2} \mathrm{O}_{2}(14$ $\mathrm{mL}, 35 \%)$ in acetic acid $(100 \mathrm{~mL})$ afforded $\mathbf{4 g}$.

Yield: $58 \%$ (10.9 g); $\mathrm{mp} 181-183^{\circ} \mathrm{C}$.

IR (ATR): 1547m, 1286s, 1256s, 1127w, 1018s, 752s, 701s, 666m cm $\mathrm{m}^{-1}$. ${ }^{1} \mathrm{H} \mathrm{NMR}\left(601 \mathrm{MHz}, \mathrm{CDCl}_{3}\right): \delta=8.76(\mathrm{~s}, 1 \mathrm{H}), 7.72(\mathrm{dd}, J=7.9,1.4 \mathrm{~Hz}$, $2 \mathrm{H}), 7.43(\mathrm{td}, J=8.4,1.5 \mathrm{~Hz}, 2 \mathrm{H}), 7.26(\mathrm{dq}, J=3.4,1.6 \mathrm{~Hz}, 2 \mathrm{H}), 7.21-$ $7.15(\mathrm{~m}, 8 \mathrm{H}), 7.01$ (td, $J=7.8,0.8 \mathrm{~Hz}, 2 \mathrm{H}), 6.96(\mathrm{~d}, J=8.4 \mathrm{~Hz}, 2 \mathrm{H})$, $3.70(\mathrm{~s}, 6 \mathrm{H})$

${ }^{13} \mathrm{C}$ NMR $\left(151 \mathrm{MHz}, \mathrm{CDCl}_{3}\right): \delta=153.46,137.02,132.68,132.40$, 130.42, 129.71, 129.27, 128.43, 125.34, 122.00, 121.51, 112.07, 55.90.

Anal. Calcd for $\mathrm{C}_{29} \mathrm{H}_{25} \mathrm{ClN}_{2} \mathrm{O}_{2}$ : C, 74.27; $\mathrm{H}, 5.37 ; \mathrm{N}, 5.97$. Found: $\mathrm{C}$, $73.71 ; \mathrm{H}, 5.25$; N, 5.78 .

\section{$\boldsymbol{N}, N^{\prime}$-Bis(2,4-dimethylphenyl)thiourea $(1 \mathrm{~h})^{9 \mathrm{~b}}$}

According to GP A, reaction of 2,4-dimethylaniline $(65.0 \mathrm{~g}, 0.5 \mathrm{~mol})$ and carbon disulfide $(30 \mathrm{~mL}, 0.5 \mathrm{~mol})$, after recrystallization from EtOH (300 $\mathrm{mL})$ afforded $\mathbf{1 h}$.
Yield: $98 \%$ ( $70.1 \mathrm{~g}$ ); mp $155-156{ }^{\circ} \mathrm{C}$ (Lit..$^{\mathrm{gb}} 214^{\circ} \mathrm{C}$ ).

IR (ATR): 3196br, 1550s, 1462s, 1263s, 1144s, 696 cm $\mathrm{cm}^{-1}$.

${ }^{1} \mathrm{H}$ NMR $\left(600 \mathrm{MHz}\right.$, DMSO- $\left.d_{6}\right): \delta=8.91(\mathrm{~s}, 2 \mathrm{H}), 7.07(\mathrm{~d}, J=7.9 \mathrm{~Hz}$, $2 \mathrm{H}), 7.01$ (s, $2 \mathrm{H}), 6.95$ (d, J = 7.8 Hz, $2 \mathrm{H}), 2.23$ (s, $6 \mathrm{H}), 2.16$ (s, $6 \mathrm{H})$.

${ }^{13} \mathrm{C}$ NMR (151 MHz, DMSO- $\left.d_{6}\right): \delta=181.91,136.21,135.77,135.39$, 131.40, 128.66, 127.22, 21.11, 18.24.

Anal. Calcd for $\mathrm{C}_{17} \mathrm{H}_{20} \mathrm{~N}_{2} \mathrm{~S}$ : C, 71.79; H, 7.09; N, 9.85; S, 11.27. Found: C, 71.15; H, 7.05; N, 9.65; S, 11.92.

\section{1,3-Bis(2,4-dimethylphenyl)-4,5-diphenylimidazolin-2-thione} (2h)

Reaction of thiourea $\mathbf{1 h}(20.2 \mathrm{~g}, 70 \mathrm{mmol})$ and benzoin $(14.85 \mathrm{~g}, 70$ $\mathrm{mmol})$ in acetic acid $(100 \mathrm{~mL})$ for $\mathbf{7} \mathbf{h}$ afforded $\mathbf{5 h}$, as a mixture of two isomers (4:3) after recrystallization from $\mathrm{CHCl}_{3} / \mathrm{EtOH}$ in isomer ratio $7: 2$.

Yield: 63\% (20.3 g); $\mathrm{mp} 235-237^{\circ} \mathrm{C}$.

IR (ATR): $1502 \mathrm{~m}, 1339 \mathrm{~s}, 757 \mathrm{~m}, 694 \mathrm{~s}, 576 \mathrm{~s} \mathrm{~cm}^{-1}$.

${ }^{1} \mathrm{H}\left(601 \mathrm{MHz}, \mathrm{CDCl}_{3}\right): \delta$ (major isomer) $=7.14(\mathrm{~d}, J=7.9 \mathrm{~Hz}, 2 \mathrm{H}), 7.10-$ $7.05(\mathrm{~m}, 2 \mathrm{H}), 7.01(\mathrm{dd}, J=10.3,4.7 \mathrm{~Hz}, 4 \mathrm{H}), 6.98(\mathrm{~d}, J=7.8 \mathrm{~Hz}, 2 \mathrm{H})$, 6.96-6.91 (m, $6 \mathrm{H}), 2.23(\mathrm{~s}, 6 \mathrm{H}), 2.07(\mathrm{~s}, 6 \mathrm{H})$.

${ }^{13} \mathrm{C}$ NMR $\left(101 \mathrm{MHz}, \mathrm{CDCl}_{3}\right): \delta=165.17,139.17,135.96,133.53$, 131.88, 130.17, 129.41, 129.04, 128.18, 128.16, 127.56, 21.28, 18.16.

Anal. Calcd for $\mathrm{C}_{31} \mathrm{H}_{28} \mathrm{~N}_{2} \mathrm{~S}$ : C, 80.83; H, 6.13; N, 6.08; S, 6.96. Found: $\mathrm{C}$, $80.54 ; \mathrm{H}, 6.15$; N, 5.96; S, 6.95 .

\section{1,3-Bis(2,4-dimethylphenyl)-4,5-diphenylimidazolium Perchlo- rate (3h)}

Reaction of imidazolinethione $\mathbf{2 h}(9.12 \mathrm{~g}, 20 \mathrm{mmol})$ and $\mathrm{H}_{2} \mathrm{O}_{2}(7 \mathrm{~mL}$, $35 \%)$ in acetic acid (50 $\mathrm{mL})$ afforded $\mathbf{3 h}$.

Yield: $51 \%$ (5.4 g); $\mathrm{mp} 262-264^{\circ} \mathrm{C}$.

IR (ATR): $1537 \mathrm{~m}, 1079 \mathrm{~s}, 822 \mathrm{~m}, 784 \mathrm{~m}, 622 \mathrm{~m} \mathrm{~cm}^{-1}$.

${ }^{1} \mathrm{H}$ NMR $\left(600 \mathrm{MHz}, 80{ }^{\circ} \mathrm{C}\right.$, DMSO- $\left.d_{6}\right): \delta=9.84(\mathrm{~s}, 1 \mathrm{H}), 7.50(\mathrm{~d}, J=$ $7.6 \mathrm{~Hz}, 2 \mathrm{H}$ ), 7.31 (ddd, $J=6.2,3.2,1.5 \mathrm{~Hz}, 2 \mathrm{H}), 7.28-7.22(\mathrm{~m}, 8 \mathrm{H})$, 7.21-7.15 (m, $4 \mathrm{H}), 2.30$ (s, $6 \mathrm{H}), 2.13(\mathrm{~s}, 6 \mathrm{H})$.

${ }^{13} \mathrm{C}$ NMR (151 MHz, $80{ }^{\circ} \mathrm{C}$, DMSO- $d_{6}$ ): $\delta=141.49,138.43,134.85$, $132.95,132.30,131.11,130.67,130.52,129.06,128.59,128.16$, $125.54,21.10,17.42$.

Anal. Calcd for $\mathrm{C}_{31} \mathrm{H}_{29} \mathrm{ClN}_{2} \mathrm{O}_{4}$ : C, 70.38; H, 5.53; N, 5.30. Found: C, 70.32; H, 5.51; N, 5.25 .

\section{1,3-Bis(2,4-dimethylphenyl)-4,5-diphenylimidazolium Chloride} (4h)

Reaction of imidazolinethione $\mathbf{2 h}(4.61 \mathrm{~g}, 10 \mathrm{mmol})$ and $\mathrm{H}_{2} \mathrm{O}_{2}(3.5 \mathrm{~mL}$, $35 \%)$ in acetic acid $(25 \mathrm{~mL})$ afforded $\mathbf{4 h}$.

Yield: $69 \%$ (3.2 g); mp $248-249{ }^{\circ} \mathrm{C}$ (dec.).

IR (ATR): 2892m, 1531s, 1443m, 1228s, 825m, 785s, 696s, 565 cm $\mathrm{m}^{-1}$. ${ }^{1} \mathrm{H}$ NMR $\left(600 \mathrm{MHz}, 80{ }^{\circ} \mathrm{C}\right.$, DMSO- $\left.d_{6}\right): \delta=9.96(\mathrm{~s}, 1 \mathrm{H}), 7.54(\mathrm{~d}, J=$ $7.8 \mathrm{~Hz}, 2 \mathrm{H}), 7.33-7.28$ (m, $2 \mathrm{H}), 7.27-7.21(\mathrm{~m}, 8 \mathrm{H}), 7.18(\mathrm{~s}, 2 \mathrm{H}), 7.16$ $(\mathrm{d}, J=8.0 \mathrm{~Hz}, 2 \mathrm{H}), 2.29(\mathrm{~s}, 6 \mathrm{H}), 2.14(\mathrm{~s}, 6 \mathrm{H})$.

${ }^{13} \mathrm{C}$ NMR $\left(151 \mathrm{MHz}, 80{ }^{\circ} \mathrm{C}\right.$, DMSO- $\left.d_{6}\right): \delta=141.40,138.52,134.84$, $132.89,132.24,131.13,130.71,130.47,129.02,128.67,128.10$, 125.60, 21.10, 17.46 .

Anal. Calcd for $\mathrm{C}_{31} \mathrm{H}_{29} \mathrm{ClN}_{2}$ : C, 80.07; $\mathrm{H}, 6.29 ; \mathrm{N}, 6.02$. Found: C, 79.66; $\mathrm{H}, 6.22 ; \mathrm{N}, 5.95$. 


\section{$N, N^{\prime}$-Bis(2-methyl-5-tert-butylphenyl)thiourea (1i)}

According to GP A, reaction of 2-methyl-5-tert-butylaniline (194.0 g, $92 \%, 1.1 \mathrm{~mol}$ ) and carbon disulfide (79 g, $1 \mathrm{~mol})$, after recrystallization from $90 \%$ EtOH (600 $\mathrm{mL})$ afforded $\mathbf{1 i}$.

Yield: $62 \%$ (125.1 g); mp 152-153 ${ }^{\circ} \mathrm{C}$.

IR (ATR): 3187br, 2962s, 1535s, 1410s, 1293s, 1258s, 1222s, 808s, 733s, $600 \mathrm{~s} \mathrm{~cm}^{-1}$.

${ }^{1} \mathrm{H}$ NMR (600 MHz, DMSO- $d_{6}$ ): $\delta=9.02(\mathrm{~s}, 2 \mathrm{H}$ ), 7.26 (s, $2 \mathrm{H}$ ), 7.15 (d, $J=8.0 \mathrm{~Hz}, 2 \mathrm{H}), 7.12$ (d, $J=8.0 \mathrm{~Hz}, 2 \mathrm{H}), 2.19(\mathrm{~s}, 6 \mathrm{H}), 1.23(\mathrm{~s}, 18 \mathrm{H})$.

${ }^{13} \mathrm{C}$ NMR (151 MHz, DMSO- $d_{6}$ ): $\delta=181.43,149.10,137.84,132.14$, 130.49, 125.20, 123.84, 34.59, 31.66, 17.88.

Anal. Calcd for $\mathrm{C}_{23} \mathrm{H}_{32} \mathrm{~N}_{2} \mathrm{~S}$ : C, 74.95; H, 8.75; N, 7.60; S, 8.70. Found: C, $74.25 ; \mathrm{H}, 8.65 ; \mathrm{N}, 7.41 ; \mathrm{S}, 9.25$.

\section{1,3-Bis(2-methyl-5-tert-butylphenyl)-4,5-di(4-methoxyphenyl)- imidazolin-2-thione (2i)}

Reaction of thiourea $1 \mathbf{i}(29.5 \mathrm{~g}, 80 \mathrm{mmol})$ and anisoin $(21.8 \mathrm{~g}, 80$ $\mathrm{mmol})$ in acetic acid $(400 \mathrm{~mL})$ for $22 \mathrm{~h}$, followed by purification by column chromatography using hexane-EtOAc (5:1) afforded $\mathbf{2} \mathbf{i}$ as a mixture of two isomers (1:1). Recrystallization from diethyl ether/hexane gave the pure isomer.

Yield: 76\% (36.8 g); $\mathrm{mp} 207-208^{\circ} \mathrm{C}$.

IR (ATR): 3000-2900br, 1505s, 1346s, 1252s, 1022m, 830s, 626m, $562 \mathrm{~s} \mathrm{~cm}^{-1}$.

${ }^{1} \mathrm{H}$ NMR $\left(600 \mathrm{MHz}\right.$, DMSO- $\left.d_{6}\right): \delta=7.22(\mathrm{dd}, J=8.0,1.6 \mathrm{~Hz}, 2 \mathrm{H}), 7.18$ $(\mathrm{s}, 2 \mathrm{H}), 7.12(\mathrm{~d}, J=8.0 \mathrm{~Hz}, 2 \mathrm{H}), 7.00(\mathrm{~d}, J=8.7 \mathrm{~Hz}, 4 \mathrm{H}), 6.63(\mathrm{~d}, J=8.7$ $\mathrm{Hz}, 4 \mathrm{H}), 3.58$ (s, $6 \mathrm{H}), 2.10$ (s, $6 \mathrm{H}), 1.18(\mathrm{~s}, 18 \mathrm{H})$.

${ }^{13} \mathrm{C}$ NMR (151 MHz, DMSO- $\left.d_{6}\right): \delta=163.58,159.52,149.46,136.29$, 133.66, 132.31, 130.66, 128.25, 127.28, 125.87, 120.91, 113.92, 55.54, 34.59, 31.50, 17.99.

Anal. Calcd for $\mathrm{C}_{39} \mathrm{H}_{44} \mathrm{~N}_{2} \mathrm{O}_{2} \mathrm{~S}$ : C, 77.45; $\mathrm{H}, 7.33 ; \mathrm{N}, 4.63 ; \mathrm{S}, 5.30$. Found: C, 77.05; H, 7.35; N, 4.45; S, 5.30.

\section{1,3-Bis(2-methyl-5-tert-butylphenyl)-4,5-di(4-methoxyphe- nyl)imidazolium perchlorate ( $3 i$ )}

$\mathrm{H}_{2} \mathrm{O}_{2}$ (52 mL, 35\%) was carefully added to the reaction solution of imidazolinethione $2 \mathbf{i}(150 \mathrm{mmol})$ in acetic acid $(250 \mathrm{~mL})$ from the previous step. The temperature was allowed to rise to $60{ }^{\circ} \mathrm{C}$ and the mixture was stirred at ambient temperature for $4 \mathrm{~h}$. All volatiles were removed by rotary evaporation and the residue was dissolved in $\mathrm{MeOH}$ $(200 \mathrm{~mL})$ and treated with a solution of $\mathrm{NaClO}_{4}(28.1 \mathrm{~g}, 0.2 \mathrm{~mol})$ dissolved in a $2: 1(\mathrm{v} / \mathrm{v})$ mixture of methanol/water $(200 \mathrm{~mL})$. A white solid precipitated. The suspension was further stirred in an ice bath and the precipitate was then filtered off and washed with water, diethyl ether and dried in vacuo to obtain product $3 \mathbf{3}$.

Yield: $70 \%$ (71.1 g); $\mathrm{mp} 264-265^{\circ} \mathrm{C}$.

IR (ATR): 1521w, 1253m, 1089s, 1027m, 837m, $621 \mathrm{~m} \mathrm{~cm}^{-1}$.

${ }^{1} \mathrm{H} \mathrm{NMR}\left(601 \mathrm{MHz}, \mathrm{CDCl}_{3}\right): \delta=8.60(\mathrm{~s}, 1 \mathrm{H}), 7.87(\mathrm{~s}, 2 \mathrm{H}), 7.39$ (dd, $J=$ $8.0,1.5 \mathrm{~Hz}, 2 \mathrm{H}), 7.17(\mathrm{~d}, J=8.1 \mathrm{~Hz}, 2 \mathrm{H}), 7.09$ (d, $J=8.1 \mathrm{~Hz}, 4 \mathrm{H}), 6.72-$ $6.67(\mathrm{~m}, 4 \mathrm{H}), 3.71(\mathrm{~s}, 6 \mathrm{H}), 2.11(\mathrm{~s}, 6 \mathrm{H}), 1.28(\mathrm{~s}, 18 \mathrm{H})$.

${ }^{13} \mathrm{C}$ NMR $\left(151 \mathrm{MHz}, \mathrm{CDCl}_{3}\right): \delta=160.51,151.77,134.76,132.76$, 132.32, 132.08, 130.61, 127.77, 126.65, 117.11, 114.13, 55.23, 34.77, 31.06, 17.02.

Anal. Calcd for $\mathrm{C}_{39} \mathrm{H}_{45} \mathrm{ClN}_{2} \mathrm{O}_{6}$ : C, 69.58; H, 6.74; N, 4.16. Found: C, 69.23; H, 6.56; N, 4.05 .
1,3-Bis(2-methyl-5-tert-butylphenyl)-4,5-di(4-methoxyphenyl)imidazolium Chloride Methanol (4i)

Reaction of imidazolinethione $2 \mathbf{i}$ ( $36.29 \mathrm{~g}, 60 \mathrm{mmol}$ ) and $\mathrm{H}_{2} \mathrm{O}_{2}(18 \mathrm{~mL}$, $35 \%)$ in acetic acid $(150 \mathrm{~mL})$ afforded $\mathbf{4 i}$. An analytically pure sample was obtained by recrystallization from $\mathrm{MeOH} / \mathrm{Et}_{2} \mathrm{O}$ at $-18{ }^{\circ} \mathrm{C}$.

Yield: $70 \%$ (26.6 g); $\mathrm{mp} 128-130{ }^{\circ} \mathrm{C}$.

IR (ATR): 2963br, 1505s, 1444m, 1247s, 1182s, 1033s, 832s, $655 \mathrm{~m}$ $\mathrm{cm}^{-1}$.

${ }^{1} \mathrm{H}$ NMR (601 MHz, $\mathrm{CDCl}_{3}$ ): $\delta=10.69$ (s, $1 \mathrm{H}$ ), 7.51 (br. s., $2 \mathrm{H}$ ), 7.37 (dd, $J=8.1,1.9 \mathrm{~Hz}, 2 \mathrm{H}), 7.21(\mathrm{~d}, J=8.1 \mathrm{~Hz}, 2 \mathrm{H}), 6.96(\mathrm{~d}, J=8.1 \mathrm{~Hz}, 4$ H), 6.74-6.67 (m, 4 H), 3.73 (s, $6 \mathrm{H}), 3.41(\mathrm{~s}, 3 \mathrm{H}), 2.23(\mathrm{~s}, 6 \mathrm{H}), 1.25$ (s, $18 \mathrm{H})$.

${ }^{13} \mathrm{C}$ NMR $\left(101 \mathrm{MHz}, \mathrm{CDCl}_{3}\right): \delta=160.51,150.86,138.17,132.22$, $131.74,131.66,131.20,127.73,125.65,117.26,114.26,55.29,50.54$ (MeOH), 34.59, 31.11, 17.50.

Anal. Calcd for $\mathrm{C}_{41} \mathrm{H}_{48} \mathrm{ClN}_{2} \mathrm{O}_{4}$ : C, 74.92; $\mathrm{H}, 7.70 ; \mathrm{N}, 4.37$. Found: C, 74.56; H, 7.71; N, 4.35 .

\section{$N, N^{\prime}$-Bis-(2-isopropylphenyl)thiourea (1j)}

According to GP B, reaction of 2-isopropylaniline $(135.0 \mathrm{~g}, 1.0 \mathrm{~mol})$ and carbon disulfide (42 g, $0.55 \mathrm{~mol})$ in diglyme $(200 \mathrm{~mL})$, after recrystallization from $\mathrm{MeOH}(800 \mathrm{~mL})$ afforded $\mathbf{1 j}$.

Yield: $56 \%$ (100.3 g); $\mathrm{mp} 157-158^{\circ} \mathrm{C}$.

IR (ATR): 3250-3050br, 1531s, 1498s, 1258s, 767m, 573s cm-1.

${ }^{1} \mathrm{H}$ NMR $\left(600 \mathrm{MHz}\right.$, DMSO- $\left.d_{6}\right): \delta=9.02(\mathrm{~s}, 2 \mathrm{H}), 7.29(\mathrm{~d}, J=7.7 \mathrm{~Hz}$, $2 \mathrm{H}$ ), 7.24-7.20 (m, $2 \mathrm{H}$ ), 7.18-7.13 (m, $4 \mathrm{H}$ ), 3.14 (hept, $J=6.9 \mathrm{~Hz}$, $2 \mathrm{H}), 1.15$ (d, $J=6.9 \mathrm{~Hz}, 12 \mathrm{H})$.

${ }^{13} \mathrm{C}$ NMR (151 MHz, DMSO- $\left.d_{6}\right): \delta=182.89,146.08,137.02,129.77$, $127.79,126.40,126.22,28.15,23.70$.

Anal. Calcd for $\mathrm{C}_{19} \mathrm{H}_{24} \mathrm{~N}_{2} \mathrm{~S}$ : C, 73.03; H, 7.74; N, 8.97; S, 10.26. Found: C, 72.54; H, 7.61; N, 8.65; S, 10.65 .

1,3-Bis-(2-isopropylphenyl)-4,5-diphenylimidazolin-2-thione (2j) Reaction of thiourea $\mathbf{1 j}$ (9.77 g, $20 \mathrm{mmol}$ ) and benzoin (16.97 g, 80 $\mathrm{mmol}$ ) in acetic acid $(50 \mathrm{~mL})$ for $17 \mathrm{~h}$ afforded $\mathbf{2 j}$ as a mixture of two isomers $(2: 1)$.

Yield: $31 \%(12.1 \mathrm{~g})$.

${ }^{1} \mathrm{H}$ NMR $\left(600 \mathrm{MHz}\right.$, DMSO- $\left.d_{6}\right): \delta$ (major isomer $)=7.37-7.30(\mathrm{~m}, 6 \mathrm{H})$, 7.23-7.18 (m, $2 \mathrm{H}$ ), 7.16-7.07 ( $\mathrm{m}, 10 \mathrm{H}), 2.79$ (hept, $J=6.8 \mathrm{~Hz}, 2 \mathrm{H}$ ), $1.19(\mathrm{~d}, J=6.8 \mathrm{~Hz}, 6 \mathrm{H}), 0.98(\mathrm{~d}, J=6.9 \mathrm{~Hz}, 6 \mathrm{H})$.

${ }^{13} \mathrm{C}$ NMR $\left(151 \mathrm{MHz}\right.$, DMSO- $\left.d_{6}\right): \delta$ (major isomer) $=166.16,146.73$, $134.97,131.21,130.65,130.08,129.05,128.94,128.51,128.25$, $127.14,126.67,28.56,24.37,23.20$.

Filtration of the component that was insoluble in hot $\mathrm{EtOH}$ gave the pure minor isomer. $\mathrm{Mp} 250-251{ }^{\circ} \mathrm{C}$.

IR (ATR): 2962w, 1490m, 1340s, 751s, 695s, 598 cm $\mathrm{cm}^{-1}$.

${ }^{1} \mathrm{H}$ NMR $\left(600 \mathrm{MHz}\right.$, DMSO- $\left.d_{6}\right): \delta$ (minor isomer $)=7.50-7.47(\mathrm{~m}, 1 \mathrm{H})$, $7.26(\mathrm{dd}, J=7.3,1.4 \mathrm{~Hz}, 1 \mathrm{H}), 7.23-7.18(\mathrm{~m}, 4 \mathrm{H}), 7.01$ (dd, $J=4.8$, $4.3 \mathrm{~Hz}, 2 \mathrm{H}), 2.62-2.54(\mathrm{~m}, 1 \mathrm{H}), 1.11(\mathrm{~d}, J=6.8 \mathrm{~Hz}, 3 \mathrm{H}), 0.61$ (d, $J=$ $6.8 \mathrm{~Hz}, 3 \mathrm{H})$

${ }^{13} \mathrm{C}$ NMR $\left(151 \mathrm{MHz}\right.$, DMSO- $\left.d_{6}\right): \delta$ (minor isomer) $=166.65,146.52$, $134.95,131.40,130.98,130.13,128.90,128.61,128.58,128.35$, 127.09, 126.71, 28.35, 24.75, 22.55.

Anal. Calcd for $\mathrm{C}_{33} \mathrm{H}_{32} \mathrm{~N}_{2} \mathrm{~S}$ : C, 81.11; H, 6.60; N, 5.73; S, 6.56. Found: C, 79.88; H, 6.65; N, 5.54; S, 7.01. 


\section{1,3-Bis(2-isopropylphenyl)-4,5-diphenylimidazolium Perchlorate} (3j)

Reaction of imidazolinethione $2 \mathbf{j}$ (12.2 g, $25 \mathrm{mmol}$ ) and $\mathrm{H}_{2} \mathrm{O}_{2}(9 \mathrm{~mL}$, $35 \%)$ in acetic acid $(100 \mathrm{~mL})$ afforded $\mathbf{3} \mathbf{j}$ as a mixture of two isomers (2:1).

Yield: $81 \%$ (11.26 g); $\mathrm{mp} 238-241^{\circ} \mathrm{C}$.

IR (ATR): $1547 \mathrm{~m}, 1445 \mathrm{~m}, 1090 \mathrm{~s}, 763 \mathrm{~s}, 703 \mathrm{~s}, 622 \mathrm{~s} \mathrm{~cm}^{-1}$.

${ }^{1} \mathrm{H}$ NMR $\left(600 \mathrm{MHz}\right.$, DMSO- $\left.d_{6}\right): \delta$ (major isomer $)=10.16(\mathrm{~s}, 1 \mathrm{H}), 7.69-$ 7.67 (m, $2 \mathrm{H}), 7.58-7.50(\mathrm{~m}, 2 \mathrm{H}), 7.33-7.20(\mathrm{~m}, 14 \mathrm{H}), 2.77$ (sept, $J=$ $6.8 \mathrm{~Hz}, 2 \mathrm{H}), 1.13(\mathrm{~d}, J=6.8 \mathrm{~Hz}, 6 \mathrm{H}), 1.00(\mathrm{~d}, J=6.8 \mathrm{~Hz}, 6 \mathrm{H})$.

${ }^{13} \mathrm{C}$ NMR (151 MHz, DMSO- $d_{6}$ ): $\delta$ (major isomer) $=145.59,138.57$, 133.07, 132.21, 131.41, 131.34, 130.59, 129.00, 128.86, 127.69, 127.57, 125.22, 28.35, 25.17, 22.62.

${ }^{1} \mathrm{H}$ NMR $\left(600 \mathrm{MHz}\right.$, DMSO- $\left.d_{6}\right): \delta$ (minor isomer) $=10.09(\mathrm{~s}, 1 \mathrm{H}), 7.88-$ 7.85 (m, $2 \mathrm{H}), 7.58-7.49$ (m, $4 \mathrm{H}), 7.49-7.44(\mathrm{~m}, 4 \mathrm{H}), 7.41-7.37$ (m, $4 \mathrm{H}), 7.33-7.20$ (m, $4 \mathrm{H}), 2.74(\mathrm{sept} J=6.8 \mathrm{~Hz}, 2 \mathrm{H}), 1.12(\mathrm{~d}, J=6.8 \mathrm{~Hz}$, $6 \mathrm{H}), 0.66(\mathrm{~d}, J=6.8 \mathrm{~Hz}, 6 \mathrm{H})$.

${ }^{13} \mathrm{C}$ NMR (151 MHz, DMSO- $\left.d_{6}\right): \delta$ (minor isomer) $=145.83,138.18$, $132.97,132.27,131.45,131.32,130.50,129.43,129.01,127.80$, 127.50, 125.50, 27.92, 25.39, 22.42.

Anal. Calcd for $\mathrm{C}_{33} \mathrm{H}_{33} \mathrm{ClN}_{2} \mathrm{O}_{4}$ : C, 70.38; H, 5.53; N, 5.30. Found: C, 70.35; H, 5.69; N, 5.12 .

\section{$N, N^{\prime}$-Bis(2-tert-butylphenyl)thiourea (1k)}

According to GP B, reaction of 2-tert-butylaniline (25.0 g, $0.168 \mathrm{~mol})$ and carbon disulfide $(7 \mathrm{~g}, 90 \mathrm{mmol})$ in diglyme $(100 \mathrm{~mL})$, after recrystallization from EtOH $(80 \mathrm{~mL})$ afforded $\mathbf{1 k}$.

Yield: $38 \%$ (10.9 g); $\mathrm{mp} 165-166^{\circ} \mathrm{C}$.

IR (ATR): 3374m, 3218br, 2961br, 1526s, 1479s, 1256s, 753s, 632s $\mathrm{cm}^{-1}$.

${ }^{1} \mathrm{H}$ NMR (600 MHz, DMSO- $d_{6}$ ): $\delta=8.91(\mathrm{~s}, 2 \mathrm{H}), 7.40-7.35(\mathrm{~m}, 2 \mathrm{H})$, 7.19-7.15 (m, $6 \mathrm{H}), 1.36(\mathrm{~s}, 18 \mathrm{H})$.

${ }^{13} \mathrm{C}$ NMR (151 MHz, DMSO- $\left.d_{6}\right): \delta=183.20,146.97,138.03,133.20$, 127.60, 127.23, 126.70, 35.33, 31.38.

Anal. Calcd for $\mathrm{C}_{21} \mathrm{H}_{28} \mathrm{~N}_{2} \mathrm{~S}$ : C, 74.07; H, 8.29; N, 8.23; S, 9.41. Found: C, 73.85; H, 8.26; N, 8.20; S, 9.48.

\section{2-[(2-tert-Butylphenyl)amino]-1,2-diphenylethanone (5)}

Reaction of thiourea $1 \mathbf{k}(11.9 \mathrm{~g}, 35 \mathrm{mmol})$ and benzoin $(7.41 \mathrm{~g}, 35$ $\mathrm{mmol})$ in acetic acid $(50 \mathrm{~mL})$ for $17 \mathrm{~h}$, after recrystallization from diethyl ether/hexane afforded $\mathbf{5}$.

Yield: $51 \%$ (6.1 g); $\mathrm{mp} 154-155^{\circ} \mathrm{C}$.

IR (ATR): 3461m, 2956br, 1683s, 1506s, 1448s, 1250s, 744s, 693s, $635 \mathrm{~s}, 595 \mathrm{~s} \mathrm{~cm}^{-1}$.

${ }^{1} \mathrm{H}$ NMR (600 MHz, DMSO- $\left.d_{6}\right): \delta=8.14(\mathrm{~d}, J=8.0 \mathrm{~Hz}, 2 \mathrm{H}), 7.61-7.54$ $(\mathrm{m}, 1 \mathrm{H}), 7.50(\mathrm{~d}, J=8.0 \mathrm{~Hz}, 2 \mathrm{H}), 7.47(\mathrm{t}, J=7.6 \mathrm{~Hz}, 2 \mathrm{H}), 7.23(\mathrm{t}, J=$ $7.6 \mathrm{~Hz}, 2 \mathrm{H}), 7.15-7.07(\mathrm{~m}, 2 \mathrm{H}), 6.89(\mathrm{t}, J=7.6 \mathrm{~Hz}, 1 \mathrm{H}), 6.72(\mathrm{~d}, J=$ $8.1 \mathrm{~Hz}, 1 \mathrm{H}), 6.53-6.47(\mathrm{~m}, 2 \mathrm{H}), 5.87(\mathrm{~d}, J=5.2 \mathrm{~Hz}, 1 \mathrm{H}), 1.45(\mathrm{~s}, 9 \mathrm{H})$.

${ }^{13} \mathrm{C}$ NMR (151 MHz, DMSO- $d_{6}$ ): $\delta=198.00,155.50,148.73,143.79$, $138.82,134.86,134.35,133.25,129.59,129.30,129.29,128.69$, 128.24, 127.15, 126.41, 117.17, 113.05, 61.88, 34.32, 30.17.

Anal. Calcd for $\mathrm{C}_{24} \mathrm{H}_{25} \mathrm{NO}$ : C, 83.93; H, 7.34; N, 4.08. Found: C, 83.65; H, 7.29; N, 4.01 .

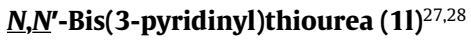

According to GP B, reaction of 3-aminopyridine (94.1 g, $1 \mathrm{~mol}$ ) and carbon disulfide $(38 \mathrm{~mL}, 0.63 \mathrm{~mol})$ in diglyme $(250 \mathrm{~mL})$, after recrystallization from $\mathrm{EtOH} / \mathrm{H}_{2} \mathrm{O}(200 / 50 \mathrm{~mL})$ afforded 11. An analytically pure sample was obtained by recrystallization from ethanol.

Yield: $35 \%$ (40.6 g); mp $178-179{ }^{\circ} \mathrm{C}$ ( Lit. $^{27} 178-180{ }^{\circ} \mathrm{C}$ ).

IR (ATR): 2971m, 2902m, 1580s, 1536s, 1414s, 1268s, 1252s, 1047s, 1024s, 1016s, 753s, 717s, 701s cm c $^{-1}$.

${ }^{1} \mathrm{H}$ NMR (601 MHz, DMSO- $d_{6}$ ): $\delta=10.08$ (s, $2 \mathrm{H}$ ), 8.63 (dd, $J=2.6$, $0.6 \mathrm{~Hz}, 2 \mathrm{H}), 8.37(\mathrm{dd}, J=4.7,1.5 \mathrm{~Hz}, 2 \mathrm{H}), 7.97(\mathrm{ddd}, J=8.2,2.6$, $1.5 \mathrm{~Hz}, 2 \mathrm{H}$ ), 7.40 (ddd, $J=8.2,4.7,0.6 \mathrm{~Hz}, 2 \mathrm{H}$ ).

${ }^{13} \mathrm{C}$ NMR $\left(151 \mathrm{MHz}\right.$, DMSO- $\left.d_{6}\right): \delta=181.47,146.04,145.99,136.56$, 132.04, 123.73.

NMR spectroscopic data of $\mathbf{1 1}$ matched those previously described. ${ }^{28}$

2-[(3-Pyridyl)amino]-1,2-diphenylethanone (6)

Reaction of thiourea 11 (34.66 g, $0.15 \mathrm{~mol}$ ) and benzoin (31.81 g, 0.15 $\mathrm{mol})$ in acetic acid $(150 \mathrm{~mL})$ for $10 \mathrm{~h}$ afforded $\mathbf{6}$. An analytically pure sample was obtained by recrystallization from $\mathrm{EtOH} / \mathrm{H}_{2} \mathrm{O}(1: 1)$.

Yield: 95\% (40.9 g); $\mathrm{mp} 141-142{ }^{\circ} \mathrm{C}$.

IR (ATR): 3326w, 2336w, 1678s, 1589s, 1578s, 1342s, 795s, 755s, 711s, 700s, 691s, 681s, 668s, 618s cm-1.

${ }^{1} \mathrm{H}$ NMR $\left(601 \mathrm{MHz}, \mathrm{CDCl}_{3}\right): \delta=8.05(\mathrm{~d}, J=2.8 \mathrm{~Hz}, 1 \mathrm{H}), 7.94-7.91(\mathrm{~m}, 2$ H), $7.86(\mathrm{dd}, J=4.6,1.3 \mathrm{~Hz}, 1 \mathrm{H}), 7.49-7.45(\mathrm{~m}, 1 \mathrm{H}), 7.39-7.34(\mathrm{~m}, 4$ H), $7.22(\mathrm{dd}, J=10.5,4.8 \mathrm{~Hz}, 2 \mathrm{H}), 7.16-7.12(\mathrm{~m}, 1 \mathrm{H}), 6.94(\mathrm{dd}, J=8.3$, $4.6 \mathrm{~Hz}, 1 \mathrm{H}), 6.84$ (ddd, $J=8.3,2.9,1.3 \mathrm{~Hz}, 1 \mathrm{H}), 5.94(\mathrm{~d}, J=6.6 \mathrm{~Hz}, 1 \mathrm{H})$, $5.46(\mathrm{~d}, J=6.4 \mathrm{~Hz}, 1 \mathrm{H})$.

${ }^{13} \mathrm{C}$ NMR $\left(151 \mathrm{MHz}, \mathrm{CDCl}_{3}\right): \delta=196.28,142.02,139.30,136.92$, $136.44,134.71,133.76,129.25,128.91,128.77,128.43,128.15$, 123.64, 119.65, 62.25 .

Anal. Calcd for $\mathrm{C}_{19} \mathrm{H}_{16} \mathrm{~N}_{2} \mathrm{O}$ : C, 79.14; H, 5.59; N, 9.72. Found: C, 79.51; H, 5.62; N, 9.81 .

\section{$\boldsymbol{N}, \boldsymbol{N}^{\prime}$-Bis(2,4,6-trimethylphenyl)thiourea $(\mathbf{1 m})^{29,30}$}

According to GP A, reaction of 2,4,6-trimethylaniline ( $65.0 \mathrm{~g}, 0.5 \mathrm{~mol}$ ) and carbon disulfide $(30 \mathrm{~mL}, 0.5 \mathrm{~mol})$, after recrystallization from EtOH (300 $\mathrm{mL})$ afforded $\mathbf{1 m}$.

Yield: 91\% (70.9 g); mp 187-189 ${ }^{\circ} \mathrm{C}$ (Lit. $175^{\circ} \mathrm{C},{ }^{9 b} 202-203{ }^{\circ} \mathrm{C}^{29}$ ).

IR (ATR): 3321m, 3250-3150br, 3100-2900br, 1519s, 1475s, 1246s, $1220 \mathrm{~s}, 854 \mathrm{~m}, 654 \mathrm{~m} \mathrm{~cm}^{-1}$.

$\left.{ }^{1} \mathrm{H} \mathrm{NMR} \mathrm{(400} \mathrm{MHz,} \mathrm{CDCl}_{3}\right): \delta=7.94(\mathrm{~s}, 1 \mathrm{H}), 6.99(\mathrm{~s}, 2 \mathrm{H}), 6.84(\mathrm{~s}, 2 \mathrm{H})$, 6.47 (s, $1 \mathrm{H}), 2.38$ (s, $6 \mathrm{H}), 2.30$ (s, $3 \mathrm{H}), 2.22(\mathrm{~s}, 3 \mathrm{H}), 2.18(\mathrm{~s}, 6 \mathrm{H})$.

${ }^{13} \mathrm{C}$ NMR $\left(101 \mathrm{MHz}, \mathrm{CDCl}_{3}\right): \delta=181.37,139.17,137.69,137.29$, $136.11,133.16,130.65,129.79,129.05,21.01,18.52,18.10$.

NMR spectroscopic data of $\mathbf{1} \mathbf{m}$ matched those previously described. ${ }^{29,30}$

\section{$\boldsymbol{N}, \boldsymbol{N}^{\prime}$-Bis(2,6-diisopropylphenyl)thiourea (1n) $)^{30,31}$}

According to GP B, reaction of 2,6-diisopropylaniline (90\%, $394 \mathrm{~g}$, $2 \mathrm{~mol}$ ) and carbon disulfide ( $91.4 \mathrm{~g}, 1.2 \mathrm{~mol}$ ) in diglyme ( $1 \mathrm{~L})$, afforded 1n.

Yield: 39\% (153.8 g); mp 223-224 ${ }^{\circ} \mathrm{C}$ (Lit. ${ }^{31} 242{ }^{\circ} \mathrm{C}$ ).

IR (ATR): 3135m, 2959s, 1523s, 1463m, 1261s, 1234s, 800s, 481m $\mathrm{cm}^{-1}$. 
${ }^{1} \mathrm{H}$ NMR $\left(601 \mathrm{MHz}, \mathrm{CDCl}_{3}\right): \delta=8.80(\mathrm{~s}, 1 \mathrm{H}), 7.31(\mathrm{t}, J=7.7 \mathrm{~Hz}, 1 \mathrm{H})$, 7.22-7.17 (m, $3 \mathrm{H}), 7.05(\mathrm{t}, J=6.0 \mathrm{~Hz}, 2 \mathrm{H}), 6.32(\mathrm{~s}, 1 \mathrm{H}), 3.34$ (hept, $J=$ $6.8 \mathrm{~Hz}, 2 \mathrm{H}), 2.96$ (hept, $J=6.8 \mathrm{~Hz}, 2 \mathrm{H}), 1.29$ (d, $J=6.9 \mathrm{~Hz}, 6 \mathrm{H}), 1.23$ $(\mathrm{d}, J=6.8 \mathrm{~Hz}, 6 \mathrm{H}), 1.18(\mathrm{~d}, J=6.8 \mathrm{~Hz}, 6 \mathrm{H}), 0.96(\mathrm{~d}, J=6.9 \mathrm{~Hz}, 6 \mathrm{H})$.

${ }^{13} \mathrm{C}$ NMR $\left(101 \mathrm{MHz}, \mathrm{CDCl}_{3}\right): \delta=182.44,148.14,146.64,132.74$, $130.60,130.11,128.81,124.23,123.62,28.89,28.58,26.01,24.43$, $23.84,22.10$

NMR spectroscopic data for 1 n matched those previously described. ${ }^{30}$

\section{Acknowledgment}

We are grateful to Prof. J. Christoffers from the Carl von Ossietzky University of Oldenburg for recording IR spectra and to Dr. O. Tok from the Institute of Inorganic Chemistry, Academy of Science of the Czech Republic for recording NMR spectra.

\section{Supporting Information}

Supporting information for this article is available online at https://doi.org/10.1055/s-0039-1690338.

\section{References}

(1) Current address: C. Azap, Chemetall GmbH, Trakehner Str. 3, 60487 Frankfurt am Main, Germany.

(2) Current address: A. Christoffers, Diapharm Analytics GmbH, Würzburger Str. 2, 26121 Oldenburg, Germany.

(3) (a) Herrmann, W. A. Kocher C Angew. Chem., Int. Ed. Engl.; 1997, 36, 2163; Angew. Chem. 1997, 109, 2256. (b) Bourissou, D.; Guerret, O.; Gabbai, F. P.; Bertrand, G. Chem. Rev. 2000, 100, 39. (c) $\mathrm{N}$-Heterocyclic Carbenes in Transition Metal Catalysis; Glorius, F., Ed.; Springer: Berlin, 2007. (d) Diez-Gonzalez, S.; Marion, N.; Nolan, S. P. Chem. Rev. 2009, 109, 3612. (e) Corberán, R.; Mas, E.; Peris-Marzá, E. Eur. J. Inorg. Chem. 2009, 1700. (f) Hopkinson, M. N.; Richter, C.; Schedler, M.; Glorius, F. Nature 2014, 510, 485. (g) N-Heterocyclic Carbenes: Effective Tools for Organometallic Synthesis; Nolan, S. P., Ed.; Wiley-VCH: Weinheim, 2014. (h) $N$-Heterocyclic Carbenes: From Laboratory Curiosities to Efficient Synthetic Tools; Diez-Gonzalez, S., Ed.; Royal Society of Chemistry: Cambridge, 2017.

(4) Occhipinti, G.; Bjørsvik, H.-R.; Jensen, V. R. J. Am. Chem. Soc. 2006, 128, 6952.

(5) (a) Lübbe, C.; Dumrath, A.; Neumann, H.; Beller, M.; Kadyrov, R. ChemCatChem 2014, 6, 105. (b) Kadyrov, R.; Azap, C.; Weidlich, S.; Wolf, D. Top. Catal. 2012, 55, 538. (c) Kadyrov, R.; Rosiak, A. Chemistry Today 2009, 27, 24.

(6) For reviews, see: (a) Jahnke, M. C.; Hahn, F. E. In N-Heterocyclic Carbenes: From Laboratory Curiosities to Efficient Synthetic Tools; Diez-Gonzalez, S., Ed.; Royal Society of Chemistry: London, 2017, 1-41. (b) Benhamou, L.; Chardon, E.; Lavigne, G.; Bellemin-Laponnaz, S.; Cesar, V. Chem. Rev. 2011, 111, 2705. (c) Zhong, R.; Lindhorst, A. C.; Groche, F. J.; Kühn, F. E. Chem. Rev. 2017, 117, 1970.

(7) For selected references, see: (a) Herrmann, W. A.; Goossen, L. J.; Artus, G. R. J.; Köcher, C. Organometallics 1997, 16, 2472. (b) Arduengo, A. J. III.; Krafczyk, R.; Schmutzler, R. Tetrahedron 1999, 55, 14523. (c) Hirano, K.; Urban, S.; Wang, C.; Glorius, F. Org. Lett. 2009, 11, 1019; and references cited therein. (d) Lv, T.; Wang, Z.; You, J.; Lan, J.; Gao, G.J. Org. Chem. 2013, 78, 5723.
(8) (a) Schönherr, H.-J.; Wanzlick, H.-W. Justus Liebigs Ann. Chem. 1970, 731, 176. (b) Schönherr, H.-J.; Wanzlick, H.-W. Chem. Ber. 1970, 103, 1037. (c) Arduengo, A. J. III.; Goerlich, J. R.; Krafczyk, R.; Marshall, W. J. Angew. Chem. Int. Ed. 1998, 37, 1963; Angew. Chem. 1998, 110, 2062. (d) Dowe, A. P.; Li, H.; Pratt, R. C.; Lohmeijer, B. G. G.; Culkin, D. A.; Waymouth, R. M.; Hedrick, J. L. Chem. Commun. 2006, 2881. (e) Ogle, J. W.; Zhang, J.; Reibenspies, J. H.; Abboud, K. A.; Miller, S. A. Org. Lett. 2008, 10, 3677. (f) Ogle, J. J. W.; Miller, S. A. Chem. Commun. 2009, 5728. (g) Mehrotra, K. N.; Singh, G. Synthesis 1980, 1001.

(9) (a) Schroeder, D. C. Chem. Rev. 1955, 55, 181. (b) Azizi, N.; Khajeh-Amiri, A.; Ghafuri, H.; Bolourtchian, M. Mol. Diversity 2011, 15, 157. (c) Li, Z.; Liu, D.; Chen, Y.; Yin, Y.; Wang, Z.; Sun, X. J. Chem. Res. 2016, 40, 515; and references cited therein.

(10) Sharma, S. Synthesis 1978, 803.

(11) (a) Dyson, G.; George, H. J. J. Chem. Soc. 1924, 1702. (b) Natarajan, A.; Guo, Y.; Arthanari, H.; Wagner, G.; Halperin, J. A.; Chorev, M. J. Org. Chem. 2005, 70, 6362. (c) Štrukil, V.; Igrc, M. D.; Fábián, L.; Eckert-Maksić, M.; Childs, S. L.; Reid, D. G.; Duer, M. J.; Halasz, I.; Mottilloe, C.; Friščić, T. Green Chem. 2012, $14,2462$.

(12) Staab, H. A.; Walther, G. Justus Liebigs Ann. Chem. 1962, 657, 98.

(13) (a) Ballabeni, M.; Ballini, R.; Bigi, F.; Maggi, R.; Parrini, M.; Predieri, G.; Sartori, G. J. Org. Chem. 1999, 64, 1029. (b) Venkatesh, P.; Pandeya, S. N. E-J. Chem. 2009, 6, 495. (c) Maddani, M. R.; Prabhu, K. R. J. Org. Chem. 2010, 75, 2327.

(14) Piel, I.; Pawelczyk, M. D.; Hirano, K.; Fröhlich, R.; Glorius, F. Eur. J. Org. Chem. 2011, 5475.

(15) (a) Biltz, H. Ber. Dtsch. Chem. Ges. 1907, 40, 4799. (b) Klüpfel, K. W.; Stumpf, H. R.; Behmenburg, H.; Neugebauer, W.; Süß, O.; Tomanek, M. German Patent Appl. DE 1060713, 1959; Chem. Abstr. 1961, 55, 20735b

(16) Grimmett, M. R. In Science of Synthesis, Vol. 12; Neier, R., Ed.; Georg Thieme Verlag: Stuttgart, 2002.

(17) Pesch, J.; Harms, K.; Bach, T. Eur. J. Org. Chem. 2004, 2025.

(18) Carpenter, M. S.; Easter, W. M.; Wood, T. F. J. Org. Chem. 1951, $16,586$.

(19) Dyson, G. M.; George, H. J.; Hunter, R. F. J. Chem. Soc. 1927, 436.

(20) Pohloudek-Fabini, R. Arch. Pharm. 1965, 298, 51.

(21) Strukil, V.; Gracin, D.; Magdysyuk, O. V.; Dinnebier, R. E.; Friscic, T. Angew. Chem. Int. Ed. 2015, 54, 8440 .

(22) Huebner, C. F.; Marsh, J. L.; Mizzoni, R. H.; Mull, R. P.; Schroeder, D. C.; Troxell, H. A.; Scholz, C. R. J. Am. Chem. Soc. 1953, 75, 2274.

(23) Pasha, M. A.; Madhusudana Reddy, M. B. Synth. Commun. 2009, 39, 2928.

(24) Natarajan, A.; Guo, Y.; Arthanari, H.; Wagner, G.; Halperin, J. A.; Chorev, M.J. Org. Chem. 2005, 70, 6362.

(25) Lippert, K. M.; Hof, K.; Gerbig, D.; Ley, D.; Hausmann, H.; Guenther, S.; Schreiner, P. R. Eur. J. Org. Chem. 2012, 5919.

(26) Kokorev, G. I.; Yambushev, F. D. Zh. Obshch. Khim. 1987, 57, 1552.

(27) Deady, L. W.; Ganame, D.; Hughes, A. B.; Quazi, N. H.; Zanatta, S. D. Aust. J. Chem. 2002, 55, 287.

(28) Katritzky, A. R.; Witek, R. W.; Rodriguez-Garcia, V.; Mohapatra, P. P.; Rogers, J. W.; Cusido, J.; Abdel-Fattah, A. A. A.; Steel, P. J. J. Org. Chem. 2005, 70, 7866.

(29) Yang, D.; Chen, Y.-C.; Zhu, N.-Y. Org. Lett. 2004, 6, 1577.

(30) Findlater, M.; Hill, N. J.; Cowley, A. H. Dalton Trans. 2008, 4419.

(31) Walter, W.; Randau, G. Justus Liebigs Ann. Chem. 1969, 722, 52. 